\title{
ELECTRON-ION RECOMBINATION RATE COEFFICIENTS AND PHOTOIONIZATION CROSS SECTIONS FOR ASTROPHYSICALLY ABUNDANT ELEMENTS. X. Ne VIII AND Ne IX FOR ULTRAVIOLET AND X-RAY MODELING
}

\author{
Sultana N. Nahar and Anil K. Pradhan \\ Department of Astronomy, The Ohio State University, Columbus, OH 43210; nahar@astronomy.ohio-state.edu \\ Received 2005 August 19; accepted 2005 September 30
}

\begin{abstract}
Results are presented for the inverse processes of photoionization and electron-ion recombination of $(h \nu+\mathrm{Ne}$ VIII $\leftrightarrow$ $\mathrm{Ne} \mathrm{IX}+e)$ and $(h \nu+\mathrm{Ne} \mathrm{IX} \leftrightarrow \mathrm{Ne} \mathrm{x}+e)$ using the self-consistent unified method. The method employs an identical wave function expansion for both photoionization and recombination, and it includes both radiative and dielectronic recombination. Total, as well as level-specific, photoionization cross sections, $\sigma_{\mathrm{PI}}(E ; n S L J)$, and recombination rate coefficients, $\alpha_{R}(T ; n S L J)$, are presented for all fine-structure levels up to $n \leq 10$. These correspond to a total of 98 bound fine-structure levels of $\mathrm{Ne}$ vIII with $1 / 2 \leq J \leq 17 / 2$, and 178 bound levels of $\mathrm{Ne}$ Ix with $0 \leq J \leq 10$. Total recombination cross sections and rates as functions of electron energy are also presented. The coupled channel wave function expansions for the core ions include 17 levels of Ne Ix and 16 levels of Ne x. Relativistic fine structure is considered through the Breit-Pauli $\boldsymbol{R}$-matrix (BPRM) method. The photoionization and recombination cross sections include important atomic effects such as radiation damping, channel coupling, and interference and should be of definitive accuracy. Level-specific $\sigma_{\mathrm{PI}}(n S L J)$ and $\alpha_{R}(T ; n S L J)$ are calculated for the first time. In addition, we describe the applicability of these comprehensive data sets to not only ionization balance and recombination-cascade models for astrophysical and laboratory plasmas, but also to (1) models of UV and X-ray lines in He-like ions from $\mathrm{C} v$ to $\mathrm{Ne}$ IX involving the $2{ }^{3} P_{0,1,2}^{o} \rightarrow 2{ }^{3} S_{1}$ allowed triplet transitions in the UV, and $2\left({ }^{1} P_{1}^{o},{ }^{3} P_{1,2}^{o},{ }^{3} S_{1}\right) \rightarrow 1{ }^{1} S_{0}$ allowed, intercombination, and forbidden transitions in the X-ray, and (2) calculation of dielectronic satellite intensities from the highly resolved resonances in the unified recombination cross sections of He-like and Li-like ions through straightforward integration over a Maxwellian or other electron distribution functions.
\end{abstract}

Subject headings: atomic data — atomic processes — line: formation — X-rays: general

\section{INTRODUCTION}

He-like ions are the most important atomic species in hightemperature astrophysical and laboratory sources (e.g., Gabriel \& Jordan 1969; Leighly et al. 1997; Lee et al. 2001; Ness et al. 2003). That is not only because He-like ions can exist at high temperatures over a wider range than any other ions, but also because a well-known system of spectral lines involving transitions from the $n=2$ levels enables a comprehensive diagnostics of temperature, density, and ionization state of the plasma (e.g., Oelgoetz \& Pradhan 2004). In particular, the ionization balance in the source requires the knowledge of ionization and electron-ion recombination rates for the He-like ionization stage and adjacent Li-like and H-like stages. As part of the present series of papers, we have been studying these three ionization stages of various astrophysically abundant elements using the powerful Breit-Pauli $\boldsymbol{R}$-matrix (BPRM) method. The BPRM method has been extended to a unified treatment of (electron+ion) recombination, self-consistently with photoionization, in an ab initio manner (e.g., Nahar \& Pradhan 1997; Nahar et al. 2000).

Although the main results presented in this paper are similar to a few earlier ones for $\mathrm{C}$ IV and $\mathrm{C} \mathrm{v}$ (Nahar et al. 2000), O VI, O VII (Nahar \& Pradhan 2003), Fe xxv and Fe xxv (Nahar et al. 2001), and Ni Xxvi and Ni xxVII (Nahar 2005), we discuss two useful extensions of these studies to (1) calculation of dielectronic satellite intensities from the unified (electron+ion) recombination cross sections that correspond directly to the observed recombination spectra (usually labeled DR spectra but of course including the RR contribution, albeit small), and (2) UV lines due to transitions among the $n=2$ triplet levels. Thus, the results in for $\mathrm{Li}$-, $\mathrm{He}-$, and $\mathrm{H}$-like ions presented in this paper and previous papers can be utilized for the analysis of not only X-ray observations from Chandra and XMM-Newton, but also some UV observations from the Far Ultraviolet Spectroscopic Explorer (FUSE). Though it is difficult to carry out simultaneous observations of emission regions in both wavelength bands, the UV and the $\mathrm{X}$-ray, a unified UV and X-ray model may still be useful in the interpretation of separate, noncontemporaneous events. The data are presented at all energies and temperatures prevalent in hightemperature sources such as active galactic nuclei, supernova remnants, hot stellar coronae, etc. (e.g., Canizares et al. 2000).

Photoionization cross sections, electron-ion recombination cross sections, and rate coefficients are obtained in the unified method for the total electron-ion recombination, accounting for both radiative and dielectronic recombination (RR and DR) processes, within the frame of the relativistic BPRM method (Nahar \& Pradhan 1992, 1994; Nahar 1996; Zhang et al. 1999). The unified method provides a single set of recombination rate coefficients taking account of both RR and DR in an ab initio manner. The aim of the present series of reports (e.g., Nahar \& Pradhan 1997, hereafter Paper I) is studying and presenting accurate atomic parameters for photoionization and total (electron+ion) recombination of elements for astrophysical models for a variety of applications.

The Li-like and He-like ions show distinctive features in both photoionization and electron-ion recombination compared to lesser ionic states of multielectron ions in (1) displaying a featureless smooth structure up to high energies when narrow and dense resonances appear in well-separated $n$-complexes of resonances, and (2) it is important to consider relativistic fine structure explicitly in order to resolve the extremely narrow resonances and to allow for radiation damping owing to high 
radiative decay rates for core transitions. As pointed out by Pradhan \& Zhang (1997) and Zhang et al. (1999), radiation damping is significant only for (electron+ion) recombination with $\mathrm{H}$-like and He-like core ions, i.e., He-like and Li-like recombined ions. Radiation damping may be neglected in (electron+ion) recombination with all other ions, since autoionization rates of $10^{13}-10^{14}$ considerably exceed core radiative rates for low- $n$ resonances; see, for example, the good agreement with experimental measurements for ions such as Ar XIV (Zhang \& Pradhan 1997) and C III (Pradhan et al. 2001; see also Mannervik et al. 1998) without any damping of such resonances. Therefore, the unified recombination rate coefficients reported by Nahar and coworkers for all other ions in this series should be the most accurate available. For example, Schippers et al. (2001; see also Pradhan et al. 2001) compared their experimentally derived rate coefficients with the unified rates for $\mathrm{C}$ III, showing agreement within experimental uncertainties of $30 \%$ at all temperatures of astrophysical importance $T>5000 \mathrm{~K}$; the unified results were obtained prior to the experiment in the first paper in this series (Nahar \& Pradhan 1997) in LS coupling with no fine structure, no radiation damping, and moderate resolution. One of the strengths of the unified method is that all of these effects can be included, with arbitrarily high resolution of total recombination cross sections, if needed, as in the present work.

In the present work, we also emphasize the relevance of levelspecific recombination to emission lines in general, in or out of ionization equilibrium. The most prominent $\mathrm{X}$-ray lines are due to transitions in He-like ions from the $n=2$ singlet and triplet levels to the ground level: $1 s 2 p\left({ }^{1} P_{1}^{o},{ }^{3} P_{2,1}^{o},{ }^{3} S_{1}\right) \rightarrow 1 s^{2}\left({ }^{1} S_{0}\right)$, labeled $w,(x, y)$, and $z$ for the allowed $\mathrm{E} 1$, intersystem (magnetic quadrupole M2 and intercombination E1), and the forbidden M1 (magnetic dipole) transitions respectively. The $w,(x, y), z$ set of lines is also referred to as " $r, i, f$ " in literature (also common, but incorrect, reference to these lines is as the "triplet" system). Dipole allowed transitions among the excited $n=2$ triplet symmetry levels lead to three strong UV lines: $2{ }^{3} P_{0,1,2}^{o} \rightarrow 2{ }^{3} S_{1}$. In the present work, and other relativistic BPRM calculations reported in this series on He-like and Li-like ions, we have computed level-specific recombination rate coefficients for all individual fine-structure levels up to $n=10$.

\section{THEORY}

Photorecombination of an incident electron with the target ion may occur through (1) nonresonant, background continuum, or radiative recombination ( $R R)$,

$$
e+\mathrm{X}^{++} \leftrightarrow h \nu+\mathrm{X}^{+},
$$

which is the inverse process of direct photoionization, or (2) a two-step recombination process via an autoionizing state, i.e., dielectronic recombination (DR):

$$
e+\mathrm{X}^{++} \leftrightarrow\left(\mathrm{X}^{+}\right)^{* *} \leftrightarrow \begin{cases}(a) & e+\mathrm{X}^{++} \\ (b) & h \nu+\mathrm{X}^{+}\end{cases}
$$

The inverse of DR is photoionization. The quasi-bound doubly excited autoionizing state leads to either $(a)$ autoionization, a radiationless transition to a lower target state with the electron going into a continuum, or $(b)$ radiative stabilization to a recombined bound state via decay of the ion core (usually to the ground state) with the electron captured. The unified method subsumes both the RR and DR processes and considers photo- ionization from and recombination into the infinity of levels of the (electron+ion) system.

Photoionization and recombination calculations are carried out in detail with autoionizing resonances in the close-coupling (CC) approximation (e.g., Seaton 1987). In CC approximation the target ion (core) is represented by an $N$-electron system and the $(N+1)$-th electron is the interacting electron. The total wave function, $\Psi(E)$, of the $(N+1)$ electron-ion system of symmetry $J \pi$ is represented in terms of an expansion of target eigenfunctions as

$$
\Psi(\text { ion }+e ; E)=A \sum_{i} \chi_{i}(\text { ion }) \theta_{i}+\sum_{j} c_{j} \Phi_{j}(\text { ion }+e),
$$

where $\chi_{i}$ is the target wave function in a specific level $J_{i} \pi_{i}$ and $\theta_{i}$ is the wave function for the $(N+1)$-th electron in a channel labeled as $S_{i} L_{i}\left(J_{i}\right) \pi_{i} k_{i}^{2} \ell_{i}(J \pi) ; k_{i}^{2}$ being its incident kinetic energy. Finally, $\Phi_{j}$ are the correlation functions of the $(N+1)$ electron system that account for short range correlation and the orthogonality between the continuum and the bound orbitals.

In relativistic BPRM calculations, developed under the Iron Project (IP; Hummer et al. 1993), the set of $S L \pi$ are recoupled for $J \pi$ levels of the (electron+ion) system, followed by diagonalization of the Hamiltonian, $H_{N+1}^{\mathrm{BP}} \Psi=E \Psi$, where the BreitPauli (BP) Hamiltonian is

$$
H_{N+1}^{\mathrm{BP}}=H_{N+1}^{\mathrm{NR}}+H_{N+1}^{\mathrm{mass}}+H_{N+1}^{\mathrm{Dar}}+H_{N+1}^{\mathrm{so}} .
$$

The first term, $H_{N+1}^{\mathrm{NR}}$, is the nonrelativistic Hamiltonian,

$$
H_{N+1}^{\mathrm{NR}}=\sum_{i=1}^{N+1}\left(-\nabla_{i}^{2}-\frac{2 Z}{r_{i}}+\sum_{j>i}^{N+1} \frac{2}{r_{i j}}\right),
$$

and the additional one-body terms are

$$
\begin{aligned}
\text { the mass correction term, } H^{\text {mass }} & =-\frac{\alpha^{2}}{4} \sum_{i} p_{i}^{4}, \\
\text { the Darwin term, } H^{\mathrm{Dar}} & =\frac{Z \alpha^{2}}{4} \sum_{i} \nabla^{2}\left(\frac{1}{r_{i}}\right), \\
\text { the spin-orbit interaction term, } H^{\mathrm{so}} & =Z \alpha^{2} \sum_{i} \frac{1}{r_{i}^{3}} \boldsymbol{l}_{i} \cdot \boldsymbol{s}_{i},
\end{aligned}
$$

respectively. The spin-orbit term splits the $L S$ term in to finestructure components.

The positive and negative energy states (eq. [4]) define continuum $\left(E=k^{2} \geq 0\right)$ or bound $(E<0)$ (electron+ion) states. The reduced matrix element for the bound-free transition, $\left\langle\Psi_{B}|| \boldsymbol{D} \| \Psi_{F}\right\rangle$, can be obtained from the continuum wave function $\left(\Psi_{F}\right)$ and the bound wave function $\left(\Psi_{B}\right)$. The quantity $\boldsymbol{D}$ is the dipole operator, $\boldsymbol{D}_{L}=\sum_{i} r_{i}$, in length form, the summation index being the number of electrons. The dipole line strength, $\boldsymbol{S}=\left|\left\langle\Psi_{B}|| \boldsymbol{D} \| \mid \Psi_{F}\right\rangle\right|^{2}$, gives the photoionization cross section,

$$
\sigma_{\mathrm{PI}}=\frac{1}{g_{i}} \frac{4 \pi^{2}}{3 c} \omega \boldsymbol{S},
$$

where $g_{i}$ is the statistical weight factor of the initial bound state. For highly charged $\mathrm{H}$ - and He-like recombining ions, the probability of radiative decay of an autoionizing state is often comparable to that of autoionization (typically $10^{12}-10^{14} \mathrm{~s}^{-1}$; 
discussed in Nahar et al. 2000). With strong dipole allowed $2 p \rightarrow 1 s$ and $1 s 2 p\left({ }^{1} P_{1}^{o}\right) \rightarrow 1 s^{2}\left({ }^{1} S_{0}\right)$ transitions (e.g., Table 2 below) autoionizing resonances are radiatively damped to a significant extent. The radiative damping effect of all near-threshold resonances, up to effective quantum number $\nu \leq 10$, are considered using a resonance fitting procedure (Sakimoto et al. 1990; Pradhan \& Zhang 1997; Zhang et al. 1999).

The photorecombination cross section, $\sigma_{\mathrm{RC}}$, is obtained from the photoionization cross section, $\sigma_{\mathrm{PI}}$, through the principle of detailed balance (Milne relation) as

$$
\sigma_{\mathrm{RC}}(\epsilon)=\frac{\alpha^{2}}{4} \frac{g_{i}}{g_{j}} \frac{(\epsilon+I)^{2}}{\epsilon} \sigma_{\mathrm{PI}}
$$

in rydberg units; $\alpha$ is the fine-structure constant, $\epsilon$ is the photoelectron energy, $g_{j}$ is the statistical weight factor of the recombined ion and $I$ is the ionization potential. The values of $\sigma_{\mathrm{RC}}$ computed from the photoionization cross sections at a sufficiently large number of energies to delineate the nonresonant background and the autoionizing resonances represent both radiative and the dielectronic recombination (RR and $\mathrm{DR}$ ) processes. In the unified treatment the partial photoionization cross sections, leaving the ion in the ground state, are calculated. It is assumed that the recombining ion is in the ground state, and recombination can take place into the ground or any of the excited recombined (electron+ion) states.

Recombination rate coefficients of individual recombined levels are obtained by convolving recombination cross sections over Maxwellian electron distribution $f(v)$ at a given temperature as

$$
\alpha_{\mathrm{RC}}(T)=\int_{0}^{\infty} v f(v) \sigma_{\mathrm{RC}} d v .
$$

Contributions from the bound levels are added for the total recombination rate coefficient, $\alpha_{\mathrm{RC}}$ and for the total recombination cross sections, $\sigma_{\mathrm{RC}}$.

The unified method divides the recombined levels into two groups: group A with $n \leq n_{0}$ and all possible fine-structure $J \pi$ symmetries, and group B with $n_{0}<n \leq \infty$, where $n_{0}$ is the typically 10 . Values of $\sigma_{\mathrm{PI}}$, of a large number of bound states (group A), which include all possible states with $n \leq n_{0} \sim 10$, are obtained as described above.

Group B levels, $n_{0}<n \leq \infty$, are treated through quantum defect theory of DR within close the coupling approximation (Nahar \& Pradhan 1992, 1994). A generally valid approximation made in recombination to group B levels is that the background contribution is negligible, and DR is the dominant process in the region below the threshold of convergence for high- $n$ resonances. To each excited threshold of the core, $J_{i} \pi_{i}$, belongs an infinite series of $(N+1)$ electron levels, $J_{i} \pi_{i} \nu \ell$, to which recombination can occur. For the high $\nu$ levels DR dominates, while the background RR is small. The contributions from these levels are added by calculating the DR collision strengths, $\Omega(\mathrm{DR})$, employing extension by Nahar \& Pradhan (1994) of the precise theory of radiation damping by Bell \& Seaton (1985):

$$
\Omega(\mathrm{DR})=\sum_{S L \pi} \sum_{n}(1 / 2)(2 S+1)(2 L+1) P_{n}^{S L \pi}(\mathrm{DR}) .
$$

where the DR probability $P_{n}^{S L \pi}$ in entrance channel $n$ is $P_{n}^{S L \pi}(\mathrm{DR})=\left(1-\boldsymbol{S}_{e e}^{\dagger} \boldsymbol{S}_{e e}\right)_{n}$ and $\boldsymbol{S}_{e e}$ is the matrix for electron scattering including radiation damping. The recombination cross sec- tion, $\sigma_{\mathrm{RC}}$ in megabarns $(\mathrm{Mb})$, is related to the collision strength, $\Omega_{\mathrm{RC}}$, as

$$
\sigma_{\mathrm{RC}}(i \rightarrow j)=\pi \Omega_{\mathrm{RC}}(i, j) /\left(g_{i} k_{i}^{2}\right)\left(a_{o}^{2} / 1 . \times 10^{-18}\right),
$$

where $k_{i}^{2}$ is the incident electron energy in rydbergs. Since $\sigma_{\mathrm{RC}}$ diverges at zero photoelectron energy, the total collision strength, $\Omega$, is used in the recombination rate calculations. The unified method is not restricted to $n=10$. It can readily be extended to higher $n$. However, a detailed calculation of BPRM photoionization cross sections of high- $n$ levels is unnecessary since they approach hydrogenic behavior. Background photoionization cross sections for the high- $n$ group B levels are computed hydrogenically, which is referred to as the "high- $n$ top-up" method (Nahar 1996).

\section{COMPUTATIONS}

The calculations for photoionization and electron-ion recombination span several stages, starting with the target wave function through configuration interaction atomic structure calculations. The computations using the $\boldsymbol{R}$-matrix package of codes start with the input of these wave functions. Some additional codes are necessary for the unified recombination and photoionization.

The wave function expansion for Ne vIII consists of 17 finestructure levels of the spectroscopic configurations $1 s^{2}, 1 s 2 s$, $1 s 2 p, 1 s 3 s, 1 s 3 p$, and $1 s 3 d$ of the target ion Ne Ix. The levels, along with their relative energies, are given in Table 1 . The set of correlation configurations in the atomic structure calculations is also given in Table 1. The orbital wave functions are optimized using the atomic structure code SUPERSTRUCTURE (Eissner et al. 1974). The second term in equation (3), representing bound state correlation functions for Ne viII, includes all possible $(N+1)$ particle configurations with 0 to maximum orbital occupancies in configurations $2 s^{2}, 2 p^{2}, 3 s^{2}, 3 p^{2}, 3 d^{2}, 4 s$, and $4 p$. The energies in Table 1 are observed values from the NIST Web site. ${ }^{1}$ Radial integrals for the partial wave expansion in equation (3) are specified for orbitals $0 \leq \ell \leq 9$, with an $\boldsymbol{R}$-matrix basis set of 40 continuum functions for Ne vIII. Computations are carried out for all angular momenta, $0 \leq L \leq 11,1 / 2 \leq J \leq 17 / 2$ for Ne vIII.

The coupled channel wave function expansion for $(e+\mathrm{Ne} \mathrm{x}) \rightarrow$ $\mathrm{Ne}$ IX consists of 16 fine-structure levels of H-like Ne x. $1 s, 2 s$, $2 p, 3 s, 3 p, 3 d, 4 s, 4 p, 4 d$, and $4 f$ (there are, of course, no correlation configurations). The orbital wave functions and level energies in Table 1 are obtained from SUPERSTRUCTURE. The bound state correlation functions included all configurations from 0 to maximum orbital occupancies for the configurations $1 s^{2}$, $2 s^{2}, 2 p^{2}, 3 s^{2}, 3 p^{2}, 3 d^{2}, 4 s^{2}, 4 p^{2}, 4 d^{2}$, and $4 f^{2}$. Radial integrals for the partial wave expansion are specified for orbitals $0 \leq \ell \leq 9$, with an $\boldsymbol{R}$-matrix basis set of 30 continuum functions for $\mathrm{Ne}$ x. Computations are carried out for all angular momenta, $0 \leq L \leq$ $14,0 \leq J \leq 10$ for Ne Ix.

Both the partial and the total photoionization cross sections are obtained for all bound levels using the BPRM $\boldsymbol{R}$-matrix codes. They are extensions of the Opacity Project codes (Berrington et al. 1987) to include relativistic effects (Scott \& Burke 1980; Scott \& Taylor 1982; Berrington et al. 1995), implemented under the Iron Project (Hummer et al. 1993). The energy levels are obtained from STGB, which are then identified using the code PRCBPID (Nahar \& Pradhan 2000). Radiation damping of resonances up to $n=10$ are included using the extended codes STGF and STGBF (Nahar \& Pradhan 1994; Zhang et al. 1999).

\footnotetext{
${ }^{1}$ See http://www.nist.gov.
} 
TABLE 1

Target Levels in the Eigenfunction Expansions of Ne viII and Ne ix

\begin{tabular}{|c|c|c|c|c|}
\hline \multirow[b]{2}{*}{ Number } & \multicolumn{2}{|c|}{$\mathrm{Ne}$ IX } & \multicolumn{2}{|c|}{$\mathrm{Ne} x$} \\
\hline & Level & $\begin{array}{c}E_{t} \\
\text { (ryd) }\end{array}$ & Level & $\begin{array}{c}E_{t} \\
\text { (ryd) }\end{array}$ \\
\hline $1 \ldots \ldots \ldots \ldots \ldots \ldots \ldots$ & $1 s^{2}\left({ }^{1} S_{0}\right)$ & 0.0 & $1 s\left({ }^{2} S_{1 / 2}\right)$ & 0.00 \\
\hline $2 \ldots \ldots \ldots \ldots \ldots \ldots$ & $1 s 2 s\left({ }^{3} S_{1}\right)$ & 66.522 & $2 p\left({ }^{2} P_{1 / 2}^{o}\right)$ & 75.0787 \\
\hline 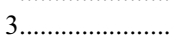 & $1 s 2 p\left({ }^{3} P_{0}^{o}\right)$ & 67.235 & $2 s\left({ }^{2} S_{1 / 2}\right)$ & 75.0802 \\
\hline 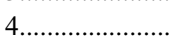 & $1 s 2 p\left({ }^{3} P_{1}^{o}\right)$ & 67.238 & $2 p\left({ }^{2} P_{3 / 2}^{o}\right)$ & 75.1122 \\
\hline $5 \ldots \ldots \ldots \ldots \ldots \ldots$ & $1 s 2 p\left({ }^{3} P_{2}^{o}\right)$ & 67.252 & $3 p\left({ }^{2} P_{1 / 2}^{o}\right)$ & 88.9941 \\
\hline 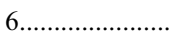 & $1 s 2 s\left({ }^{1} S_{0}\right)$ & 67.276 & $3 s\left({ }^{2} S_{1 / 2}\right)$ & 88.9945 \\
\hline 7.......................... & $1 s 2 p\left({ }^{1} P_{1}^{o}\right)$ & 67.767 & $3 d\left({ }^{2} D_{3 / 2}\right)$ & 89.0040 \\
\hline 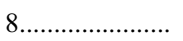 & $1 s 3 s\left({ }^{3} S_{1}\right)$ & 78.579 & $3 p\left({ }^{2} P_{3 / 2}^{o}\right)$ & 89.0040 \\
\hline 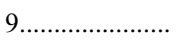 & $1 s 3 p\left({ }^{3} P_{0}\right)$ & 78.774 & $3 d\left({ }^{2} D_{5 / 2}\right)$ & 89.0073 \\
\hline $10 \ldots \ldots \ldots \ldots \ldots \ldots$ & $1 s 3 p\left({ }^{3} P_{1}^{o}\right)$ & 78.774 & $4 p\left({ }^{2} P_{1 / 2}^{o}\right)$ & 93.8631 \\
\hline $11 \ldots \ldots \ldots \ldots \ldots . .$. & $1 s 3 s\left({ }^{1} S_{0}\right)$ & 78.778 & $4 s\left({ }^{2} S_{1 / 2}\right)$ & 93.8634 \\
\hline $12 \ldots \ldots \ldots \ldots \ldots \ldots$ & $1 s 3 p\left({ }^{3} P_{2}^{o}\right)$ & 78.779 & $4 d\left({ }^{2} D_{3 / 2}\right)$ & 93.8673 \\
\hline $13 \ldots \ldots \ldots \ldots \ldots \ldots$ & $1 s 3 d\left({ }^{1} D_{1}\right)$ & 78.888 & $4 p\left({ }^{2} P_{3 / 2}^{o}\right)$ & 93.8673 \\
\hline $14 \ldots \ldots \ldots \ldots \ldots . .$. & $1 s 3 d\left({ }^{3} D_{2}\right)$ & 78.889 & $4 f\left({ }^{2} F_{5 / 2}^{o}\right)$ & 93.8687 \\
\hline $15 \ldots \ldots \ldots \ldots \ldots \ldots$ & $1 s 3 d\left({ }^{3} D_{2}\right)$ & 78.890 & $4 d\left({ }^{2} D_{5 / 2}\right)$ & 93.8687 \\
\hline $16 \ldots \ldots \ldots \ldots \ldots \ldots$ & $1 s 3 p\left({ }^{1} P_{1}^{o}\right)$ & 78.921 & $4 f\left({ }^{2} F_{7 / 2}^{o}\right)$ & 93.8694 \\
\hline $17 \ldots \ldots \ldots \ldots \ldots \ldots .$. & $1 s 3 d\left({ }^{1} D_{2}\right)$ & 78.938 & & \\
\hline
\end{tabular}

Note.-Ne IX correlation configurations: $2 s^{2}, 2 p^{2}, 3 s^{2}, 3 p^{2}, 3 d^{2}, 2 s 2 p$, $2 s 3 s, 2 s 3 p, 2 s 3 d, 2 s 4 s, 2 s 4 p, 2 p 3 s, 2 p 3 p, 2 p 3 d, 2 p 4 s$, and $2 p 4 p$.

The program PBPRAD is used to extend the total photoionization cross sections in the high-energy region beyond the highest threshold in target ion by a "tail" using a fitting formula, $\sigma_{\mathrm{PI}}^{0}\left(E^{0} / E\right)^{m}$, where $E^{0}$ is the last tabulated energy above all target thresholds and $-1 \geq m \geq-3$, as described in Nahar \& Pradhan (1994). For the Kramers fit at very high energies, $m=-3$.

Coupled channel calculations for $\sigma_{\mathrm{PI}}$ include both the background and the resonance structures due to doubly excited autoionizing states in the cross sections. It is necessary to use a very fine energy mesh in order to delineate the resonance structures. The BPRM calculations are carried out for each total angular momentum symmetry $J \pi$, corresponding to a set of fine-structure target levels $J_{t}$.

In the higher energy region, $\nu_{0}<\nu \leq \infty$ below each target threshold where the resonances are narrow and dense, and the background is negligible, we compute detailed and resonanceaveraged DR cross sections (Bell \& Seaton 1985; Nahar \& Pradhan 1994). The required radiative decay rates to the ground level for each core ion, $\mathrm{Ne}$ Ix and $\mathrm{Ne} \mathrm{x}$, are given in Table 2. The DR collision strengths are obtained using the extensions of the $\boldsymbol{R}$-matrix asymptotic region code STGF (Nahar \& Pradhan 1994; Zhang et al. 1999).

Level-specific recombination cross sections, $\sigma_{\mathrm{RC}}(i)$, into various bound levels $i \equiv n(S L J)$ of the recombined (electron+ion) system, are obtained from partial photoionization cross sections $\sigma_{\mathrm{PI}}(i, g)$ of the level $i$ into the ground level $g$ of the recombining ion. These detailed photorecombination cross sections are calculated in the energy region from the threshold energy up to $E\left(\nu=\nu_{0} \approx 10.0\right)$, where $\nu$ is the effective quantum number relative to the target level of the recombining ion. The resonances up to $\nu \leq \nu_{0}$ are delineated with a fine energy mesh. The electrons in this energy range generally recombine to a large number of final (electron+ion) levels. The level-specific rates are obtained for energies going up to infinity. Recombination cross sections are computed for all coupled symmetries and levels and summed to obtain the total $\sigma_{\mathrm{RC}}$ using the program PBPRRC (Nahar et al. 2000).

The program PBPRRC sums up the level-specific rates, which is added to the contributions from the resonant high- $n$ DR, from
TABLE 2

Radiative Decay Rates, $A_{j i}$ For Allowed Transitions to the Ground Level, $1 s^{2}{ }^{1} S_{0}$ FOR Ne IX AND $1 s^{2} S_{1 / 2}$ FOR $\mathrm{Ne} \mathrm{X}$

\begin{tabular}{|c|c|}
\hline Target Level & $\begin{array}{c}A_{f i} \\
\left(\mathrm{~s}^{-1}\right)\end{array}$ \\
\hline \multicolumn{2}{|l|}{ Ne Ix: GD $-1 s^{2}{ }^{1} S_{0}$} \\
\hline $1 s 2 p\left({ }^{3} P_{3}^{o}\right) .$. & $4.57(9)$ \\
\hline $1 s 2 p\left({ }^{3} P_{1}^{o}\right)$ & $8.88(12)$ \\
\hline $1 s 3 p\left({ }^{3} P_{3}^{o}\right)$ & $1.32(9)$ \\
\hline $1 s 3 p\left({ }^{3} P_{1}^{o}\right)$ & $2.57(12)$ \\
\hline \multicolumn{2}{|l|}{ Ne $\mathrm{x}: \mathrm{GD}-1 s^{2} S^{1 / 2}$} \\
\hline $2 p\left({ }^{2} P_{1 / 2}^{o}\right)$ & $6.26(12)$ \\
\hline 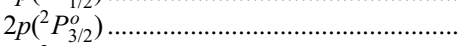 & $6.26(12)$ \\
\hline $3 p\left({ }^{2} P_{1 / 2}^{o}\right)$ & $1.65(12)$ \\
\hline $3 p\left({ }^{2} P_{3 / 2}^{o}\right)$ & $1.66(12)$ \\
\hline $4 p\left({ }^{2} P_{1 / 2}^{o}\right)$ & $6.61(11)$ \\
\hline 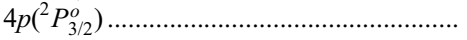 & $6.67(11)$ \\
\hline
\end{tabular}

resonances with $\nu_{0}<\nu<\infty$, to obtain total recombination rates. As an additional check on the numerical calculations, the total recombination rate coefficients, $\alpha_{R}$, are also calculated from the total recombination collision strength, $\Omega_{\mathrm{RC}}$, obtained from all the photoionization cross sections, and the DR collision strengths. The agreement between the two numerical approaches is within a few percent.

The background (nonresonant) contribution from the high- $n$ states $(10<n \leq \infty)$ to total recombination is also included as the "top-up" part, computed in the hydrogenic approximation (Nahar 1996). This contribution is important at low temperatures as the recombination rate is dominated by the RR, but negligible at high temperatures.

\section{RESULTS AND DISCUSSION}

The inverse processes of photoionization and recombination of $(\mathrm{Ne} \mathrm{VIII}+h \nu \leftrightarrow \mathrm{Ne} \mathrm{IX}+e)$ and $(\mathrm{Ne} \mathrm{IX}+h \nu \leftrightarrow \mathrm{Ne} \mathrm{x}+e)$ are studied in detail. Total recombination rate coefficients for the hydrogenic $\mathrm{Ne} x$ are also presented along with those of Ne VIII and Ne IX in Table 3 for completeness. (We note in passing that the present results for photoionization and recombination for $\mathrm{Ne}$ vIII are self-consistent with those of the oscillator strengths obtained earlier [Nahar 2002], since they are obtained using the same wave function expansion.)

Both the total and the partial photoionization cross sections including the autoionizing resonances are presented for Ne VIII and $\mathrm{Ne}$ IX for the first time and are available electronically. The total photoionization cross sections correspond to the residual core ion in various excited levels; these data are needed in spectral models and ionization balance calculations. The partial cross sections for photoionization into the ground level of the residual ion are needed to calculate the recombination rate coefficients as in the present work and may also be applicable to some NLTE models.

Total unified recombination data are computed (and are available electronically) several different ways. The recombination collision strengths $\left(\Omega_{\mathrm{RC}}\right)$, the cross sections $\left(\sigma_{\mathrm{RC}}\right)$, and the recombination rate coefficient $\left[\alpha_{\mathrm{RC}}(E)\right]$ are tabulated as a function of the photoelectron energy for Ne VIII and Ne IX, while the total unified recombination rate coefficient is a function of temperature. Values of $\alpha_{\mathrm{RC}}(T)$ are computed in two different ways to 
TABLE 3

Total Recombination Rate Coefficients $\alpha_{R}(T)$ for Ne vili, Ne ix, and Ne x

\begin{tabular}{|c|c|c|c|}
\hline \multirow{2}{*}{$\begin{array}{c}\log _{10} T \\
(\mathrm{~K})\end{array}$} & \multicolumn{3}{|c|}{$\alpha_{R}\left(\mathrm{~cm}^{3} \mathrm{~s}^{-1}\right)$} \\
\hline & $\mathrm{Ne}$ VIII & $\mathrm{Ne}$ Ix & $\mathrm{Ne} x$ \\
\hline 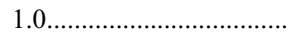 & $2.59 \mathrm{E}-09$ & $3.49 \mathrm{E}-09$ & $4.61 \mathrm{E}-09$ \\
\hline $1.1 \ldots \ldots+\ldots$ & $2.29 \mathrm{E}-09$ & $3.08 \mathrm{E}-09$ & $4.07 \mathrm{E}-09$ \\
\hline 1.2 & $2.02 \mathrm{E}-09$ & $2.72 \mathrm{E}-09$ & $3.60 \mathrm{E}-09$ \\
\hline 1.3 & $1.78 \mathrm{E}-09$ & $2.40 \mathrm{E}-09$ & $3.18 \mathrm{E}-09$ \\
\hline 1.4 & $1.56 \mathrm{E}-09$ & $2.12 \mathrm{E}-09$ & $2.81 \mathrm{E}-09$ \\
\hline 1.5 & $1.38 \mathrm{E}-09$ & $1.86 \mathrm{E}-09$ & $2.48 \mathrm{E}-09$ \\
\hline 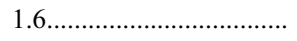 & $1.21 \mathrm{E}-09$ & $1.64 \mathrm{E}-09$ & $2.18 \mathrm{E}-09$ \\
\hline 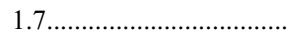 & $1.06 \mathrm{E}-09$ & $1.44 \mathrm{E}-09$ & $1.92 \mathrm{E}-09$ \\
\hline 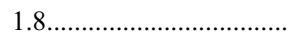 & $9.30 \mathrm{E}-10$ & $1.27 \mathrm{E}-09$ & $1.69 \mathrm{E}-09$ \\
\hline $1.9 \ldots$. & $8.15 \mathrm{E}-10$ & $1.11 \mathrm{E}-09$ & $1.49 \mathrm{E}-09$ \\
\hline $2.0 \ldots \ldots \ldots$ & $7.13 \mathrm{E}-10$ & $9.76 \mathrm{E}-10$ & $1.31 \mathrm{E}-09$ \\
\hline $2.1 \ldots \ldots$. & $6.24 \mathrm{E}-10$ & $8.56 \mathrm{E}-10$ & $1.15 \mathrm{E}-09$ \\
\hline $2.2 \ldots \ldots \ldots \ldots-1$ & $5.45 \mathrm{E}-10$ & $7.49 \mathrm{E}-10$ & $1.01 \mathrm{E}-09$ \\
\hline 2.3 & $4.76 \mathrm{E}-10$ & $6.55 \mathrm{E}-10$ & $8.82 \mathrm{E}-10$ \\
\hline $2.4 \ldots \ldots \ldots-1$ & $4.15 \mathrm{E}-10$ & $5.73 \mathrm{E}-10$ & $7.73 \mathrm{E}-10$ \\
\hline $2.5 \ldots \ldots \ldots$ & $3.62 \mathrm{E}-10$ & $5.01 \mathrm{E}-10$ & $6.77 \mathrm{E}-10$ \\
\hline $2.6 \ldots \ldots .$. & $3.16 \mathrm{E}-10$ & $4.37 \mathrm{E}-10$ & $5.92 \mathrm{E}-10$ \\
\hline $2.7 \ldots \ldots \ldots \ldots$ & $2.75 \mathrm{E}-10$ & $3.82 \mathrm{E}-10$ & $5.18 \mathrm{E}-10$ \\
\hline 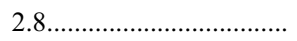 & $2.39 \mathrm{E}-10$ & $3.33 \mathrm{E}-10$ & $4.53 \mathrm{E}-10$ \\
\hline 2.9 & $2.08 \mathrm{E}-10$ & $2.90 \mathrm{E}-10$ & $3.95 \mathrm{E}-10$ \\
\hline $3.0 \ldots \ldots \ldots \ldots \ldots \ldots \ldots \ldots \ldots \ldots$ & $1.81 \mathrm{E}-10$ & $2.53 \mathrm{E}-10$ & $3.45 \mathrm{E}-10$ \\
\hline 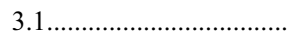 & $1.57 \mathrm{E}-10$ & $2.20 \mathrm{E}-10$ & $3.01 \mathrm{E}-10$ \\
\hline 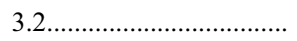 & $1.36 \mathrm{E}-10$ & $1.91 \mathrm{E}-10$ & $2.62 \mathrm{E}-10$ \\
\hline 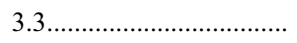 & $1.18 \mathrm{E}-10$ & $1.66 \mathrm{E}-10$ & $2.29 \mathrm{E}-10$ \\
\hline $3.4 \ldots \ldots \ldots-\ldots$ & $1.02 \mathrm{E}-10$ & $1.45 \mathrm{E}-10$ & $1.99 \mathrm{E}-10$ \\
\hline $3.5 \ldots \ldots$ & $8.85 \mathrm{E}-11$ & $1.26 \mathrm{E}-10$ & $1.74 \mathrm{E}-10$ \\
\hline 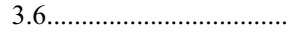 & $7.65 \mathrm{E}-11$ & $1.09 \mathrm{E}-10$ & $1.51 \mathrm{E}-10$ \\
\hline $3.7 \ldots \ldots$ & $6.61 \mathrm{E}-11$ & $9.44 \mathrm{E}-11$ & $1.31 \mathrm{E}-10$ \\
\hline 3.8 & $5.71 \mathrm{E}-11$ & $8.18 \mathrm{E}-11$ & $1.14 \mathrm{E}-10$ \\
\hline 3.9 & $4.92 \mathrm{E}-11$ & $7.09 \mathrm{E}-11$ & $9.92 \mathrm{E}-11$ \\
\hline $4.0 \ldots \ldots \ldots \ldots \ldots \ldots .$. & $4.24 \mathrm{E}-11$ & $6.13 \mathrm{E}-11$ & $8.61 \mathrm{E}-11$ \\
\hline 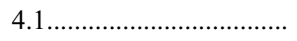 & $3.65 \mathrm{E}-11$ & $5.29 \mathrm{E}-11$ & $7.47 \mathrm{E}-11$ \\
\hline 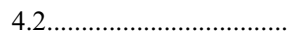 & $3.14 \mathrm{E}-11$ & $4.58 \mathrm{E}-11$ & $6.46 \mathrm{E}-11$ \\
\hline $4.3 \ldots \ldots$. & $2.69 \mathrm{E}-11$ & $3.95 \mathrm{E}-11$ & $5.62 \mathrm{E}-11$ \\
\hline 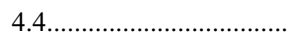 & $2.31 \mathrm{E}-11$ & $3.40 \mathrm{E}-11$ & $4.85 \mathrm{E}-11$ \\
\hline $4.5 \ldots \ldots \ldots$ & $1.98 \mathrm{E}-11$ & $2.93 \mathrm{E}-11$ & $4.20 \mathrm{E}-11$ \\
\hline 4.6 & $1.69 \mathrm{E}-11$ & $2.52 \mathrm{E}-11$ & $3.63 \mathrm{E}-11$ \\
\hline $4.7 \ldots \ldots \ldots-1$ & $1.44 \mathrm{E}-11$ & $2.17 \mathrm{E}-11$ & $3.13 \mathrm{E}-11$ \\
\hline 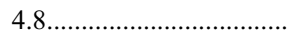 & $1.23 \mathrm{E}-11$ & $1.86 \mathrm{E}-11$ & $2.70 \mathrm{E}-11$ \\
\hline 4.9 & $1.05 \mathrm{E}-11$ & $1.60 \mathrm{E}-11$ & $2.33 \mathrm{E}-11$ \\
\hline 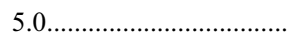 & $8.87 \mathrm{E}-12$ & $1.37 \mathrm{E}-11$ & $2.00 \mathrm{E}-11$ \\
\hline 5.1 & $7.52 \mathrm{E}-12$ & $1.17 \mathrm{E}-11$ & $1.72 \mathrm{E}-11$ \\
\hline $5.2 \ldots \ldots \ldots \ldots \ldots \ldots \ldots \ldots . .$. & $6.36 \mathrm{E}-12$ & $1.00 \mathrm{E}-11$ & $1.48 \mathrm{E}-11$ \\
\hline $5.3 \ldots \ldots \ldots \ldots \ldots \ldots \ldots \ldots . .$. & $5.36 \mathrm{E}-12$ & $8.56 \mathrm{E}-12$ & $1.27 \mathrm{E}-11$ \\
\hline 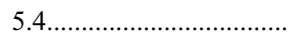 & $4.51 \mathrm{E}-12$ & $7.30 \mathrm{E}-12$ & $1.09 \mathrm{E}-11$ \\
\hline 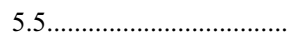 & $3.79 \mathrm{E}-12$ & $6.21 \mathrm{E}-12$ & $9.30 \mathrm{E}-12$ \\
\hline 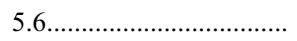 & $3.17 \mathrm{E}-12$ & $5.28 \mathrm{E}-12$ & $7.95 \mathrm{E}-12$ \\
\hline 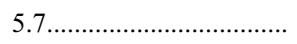 & $2.64 \mathrm{E}-12$ & $4.48 \mathrm{E}-12$ & $6.78 \mathrm{E}-12$ \\
\hline $5.8 \ldots \ldots-1$. & $2.20 \mathrm{E}-12$ & $3.79 \mathrm{E}-12$ & $5.77 \mathrm{E}-12$ \\
\hline 5.9 & $1.83 \mathrm{E}-12$ & $3.21 \mathrm{E}-12$ & $4.91 \mathrm{E}-12$ \\
\hline 6.0 & $1.52 \mathrm{E}-12$ & $2.71 \mathrm{E}-12$ & $4.16 \mathrm{E}-12$ \\
\hline 6.1 & $1.28 \mathrm{E}-12$ & $2.30 \mathrm{E}-12$ & $3.53 \mathrm{E}-12$ \\
\hline 6.2 & $1.14 \mathrm{E}-12$ & $1.99 \mathrm{E}-12$ & $2.97 \mathrm{E}-12$ \\
\hline 6.3 & $1.12 \mathrm{E}-12$ & $1.80 \mathrm{E}-12$ & $2.52 \mathrm{E}-12$ \\
\hline $6.4 \ldots \ldots \ldots \ldots \ldots \ldots \ldots .$. & $1.22 \mathrm{E}-12$ & $1.75 \mathrm{E}-12$ & $2.11 \mathrm{E}-12$ \\
\hline 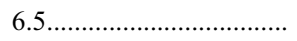 & $1.39 \mathrm{E}-12$ & $1.81 \mathrm{E}-12$ & $1.77 \mathrm{E}-12$ \\
\hline 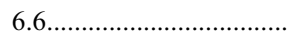 & $1.55 \mathrm{E}-12$ & $1.91 \mathrm{E}-12$ & $1.48 \mathrm{E}-12$ \\
\hline $6.7 \ldots$ & $1.64 \mathrm{E}-12$ & $1.98 \mathrm{E}-12$ & $1.23 \mathrm{E}-12$ \\
\hline $6.8 \ldots \ldots \ldots \ldots$. & $1.64 \mathrm{E}-12$ & $1.98 \mathrm{E}-12$ & $1.02 \mathrm{E}-12$ \\
\hline 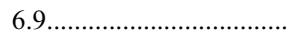 & $1.54 \mathrm{E}-12$ & $1.88 \mathrm{E}-12$ & $8.44 \mathrm{E}-13$ \\
\hline 7.0 & $1.38 \mathrm{E}-12$ & $1.71 \mathrm{E}-12$ & $6.95 \mathrm{E}-13$ \\
\hline 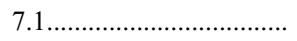 & $1.18 \mathrm{E}-12$ & $1.48 \mathrm{E}-12$ & $5.70 \mathrm{E}-13$ \\
\hline $7.2 \ldots \ldots \ldots-1$ & $9.74 \mathrm{E}-13$ & $1.25 \mathrm{E}-12$ & $4.62 \mathrm{E}-13$ \\
\hline
\end{tabular}

TABLE 3-Continued

\begin{tabular}{|c|c|c|c|}
\hline \multirow{2}{*}{$\begin{array}{c}\log _{10} T \\
(\mathrm{~K})\end{array}$} & \multicolumn{3}{|c|}{$\alpha_{R}\left(\mathrm{~cm}^{3} \mathrm{~s}^{-1}\right)$} \\
\hline & $\mathrm{Ne}$ VIII & $\mathrm{Ne}$ Ix & $\mathrm{Ne} x$ \\
\hline 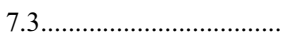 & $7.80 \mathrm{E}-13$ & $1.02 \mathrm{E}-12$ & $3.76 \mathrm{E}-13$ \\
\hline 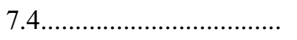 & $6.11 \mathrm{E}-13$ & $8.07 \mathrm{E}-13$ & $3.02 \mathrm{E}-13$ \\
\hline 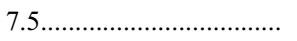 & $4.69 \mathrm{E}-13$ & $6.30 \mathrm{E}-13$ & $2.42 \mathrm{E}-13$ \\
\hline 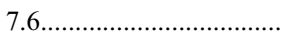 & $3.55 \mathrm{E}-13$ & $4.84 \mathrm{E}-13$ & $1.93 \mathrm{E}-13$ \\
\hline 7.7. & $2.66 \mathrm{E}-13$ & $3.67 \mathrm{E}-13$ & $1.53 \mathrm{E}-13$ \\
\hline $7.8 \ldots$. & $1.97 \mathrm{E}-13$ & $2.76 \mathrm{E}-13$ & $1.20 \mathrm{E}-13$ \\
\hline $7.9 \ldots \ldots$ & $1.45 \mathrm{E}-13$ & $2.06 \mathrm{E}-13$ & $9.39 \mathrm{E}-14$ \\
\hline 8.0 & $1.06 \mathrm{E}-13$ & $1.52 \mathrm{E}-13$ & $7.30 \mathrm{E}-14$ \\
\hline $8.1 \ldots \ldots \ldots \ldots \ldots \ldots \ldots \ldots \ldots .$. & $7.68 \mathrm{E}-14$ & $1.12 \mathrm{E}-13$ & $5.65 \mathrm{E}-14$ \\
\hline $8.2 \ldots \ldots \ldots \ldots \ldots \ldots$ & $5.57 \mathrm{E}-14$ & $8.26 \mathrm{E}-14$ & $4.32 \mathrm{E}-14$ \\
\hline $8.3 \ldots \ldots \ldots \ldots \ldots \ldots$ & $4.02 \mathrm{E}-14$ & $6.05 \mathrm{E}-14$ & $3.32 \mathrm{E}-14$ \\
\hline 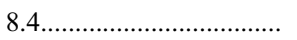 & $2.90 \mathrm{E}-14$ & $4.42 \mathrm{E}-14$ & $2.51 \mathrm{E}-14$ \\
\hline 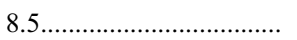 & $2.08 \mathrm{E}-14$ & $3.22 \mathrm{E}-14$ & $1.90 \mathrm{E}-14$ \\
\hline 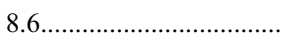 & $1.50 \mathrm{E}-14$ & $2.35 \mathrm{E}-14$ & $1.43 \mathrm{E}-14$ \\
\hline $8.7 \ldots$ & $1.07 \mathrm{E}-14$ & $1.71 \mathrm{E}-14$ & $1.07 \mathrm{E}-14$ \\
\hline 8.8 & $7.69 \mathrm{E}-15$ & $1.24 \mathrm{E}-14$ & $7.99 \mathrm{E}-15$ \\
\hline 8.9 & $5.51 \mathrm{E}-15$ & $9.00 \mathrm{E}-15$ & $5.93 \mathrm{E}-15$ \\
\hline 9.0 & $3.94 \mathrm{E}-15$ & $6.52 \mathrm{E}-15$ & $4.39 \mathrm{E}-15$ \\
\hline
\end{tabular}

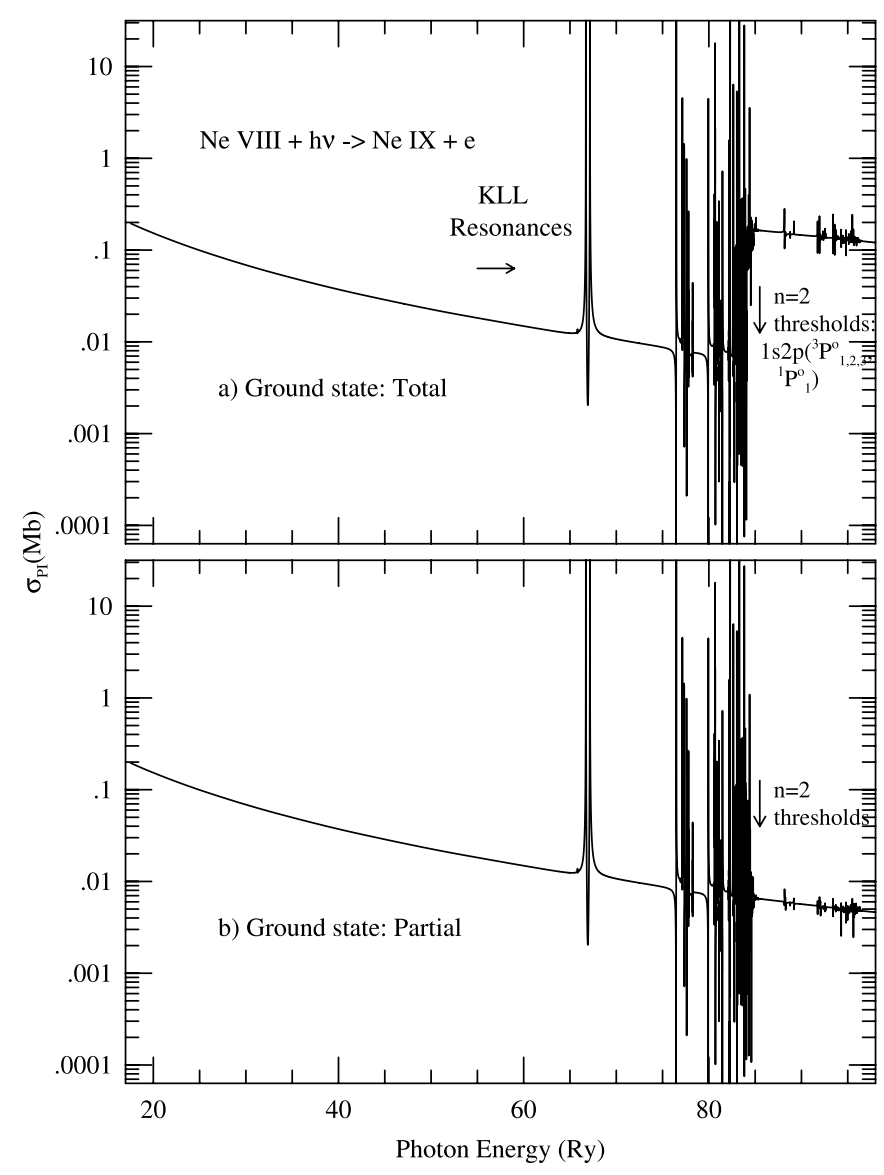

FIG. 1.-Photoionization cross sections $\left(\sigma_{\mathrm{PI}}\right)$ of the ground level $1 s^{2} 2 s\left({ }^{2} S_{1 / 2}\right)$ of Ne VIII: (a) Total cross section; the large jump around $n=2$ thresholds ( $\sim 85$ ryd) is the $K$-shell ionization edge $\left(h \nu+1 s^{2} 2 l \rightarrow 1 s 2 l+e\right.$ ). (b) Partial cross section for ionization into the ground level $1 s^{2}\left({ }^{1} S_{0}\right)$ of core Ne Ix; note that the jump no longer exists and the cross section is continuous across the $n=2$ thresholds. 


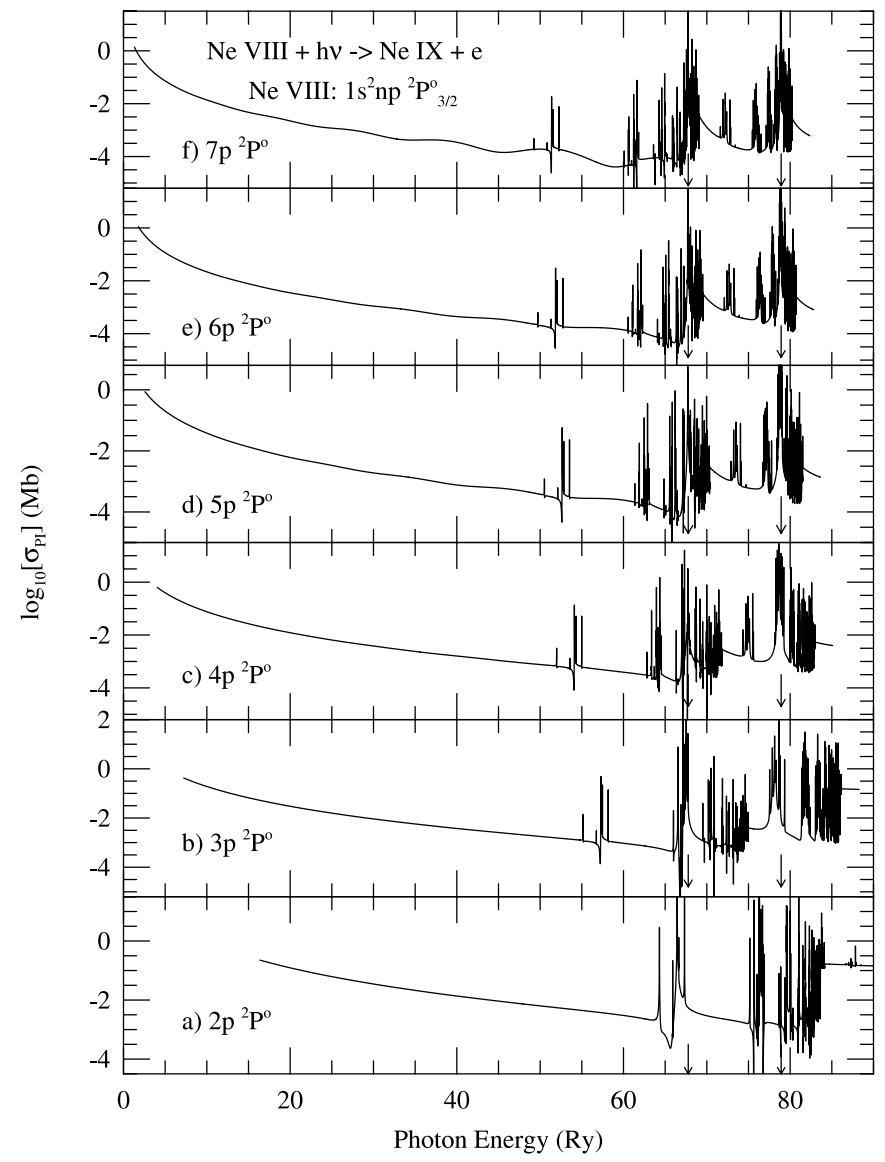

FIG. 2.-Photoionization cross sections of the Rydberg series of levels, $1 s^{2} n p\left({ }^{2} P_{3 / 2}^{o}\right.$ ) with $2 \leq n \leq 7$, of Ne VIII. Prominent PEC (photoexcitation-ofcore) resonances are seen (denoted by arrows) at about 67.75 ryd due to excited $n=2$ core levels $1 s 2 p{ }^{3} P_{1}^{o},{ }^{1} P_{1}^{o}$ and at about 78.90 ryd due to $n=3$ core levels $1 s 3 p^{3} P_{1}^{o},{ }^{1} P_{1}^{o}$.

enable numerical checks: (1) from the sum of the level-specific rate coefficients and the high- $n$ DR contribution and (2) from the total collision strengths calculated from photoionization cross sections directly, plus the DR contribution. The differences between the two are typically within a few percent, thus providing numerical and self-consistency checks, particularly on the resolution of resonances.

Level-specific recombination rate coefficients, $\alpha_{i}$, for Ne VIII and $\mathrm{Ne}$ IX are presented for all levels $(n S L J, n \leq 10)$. Calculations of the recombination-cascade contributions for important lines require accurate atomic parameters for fine-structure levels up to fairly high $n$ levels, as presented in this report. The levelspecific rate coefficients are obtained for the first time (currently available data exist only for total RR and DR rates). Some important features in photoionization and electron-ion recombination for each ion are discussed separately below.

\subsection{Ne VIII}

A total of 98 bound levels are found for Ne vIII with $n \leq 10$, $0 \leq l \leq 9,0 \leq L \leq 11$, and total angular momentum of $1 / 2 \leq$ $J \leq 17 / 2$ (Nahar 2002).

\subsubsection{Photoionization}

Cross sections $\left(\sigma_{\mathrm{PI}}\right)$ for both total and partial photoionization obtained for all 98 bound levels of Ne vIII. Figures $1 a$ and $1 b$ show the ground state photoionization cross section for $\mathrm{Ne}$ VIII $\left(1 s^{2} 2 s^{2} S_{1 / 2}\right)$. Figure $1 a$ presents the total photoionization cross

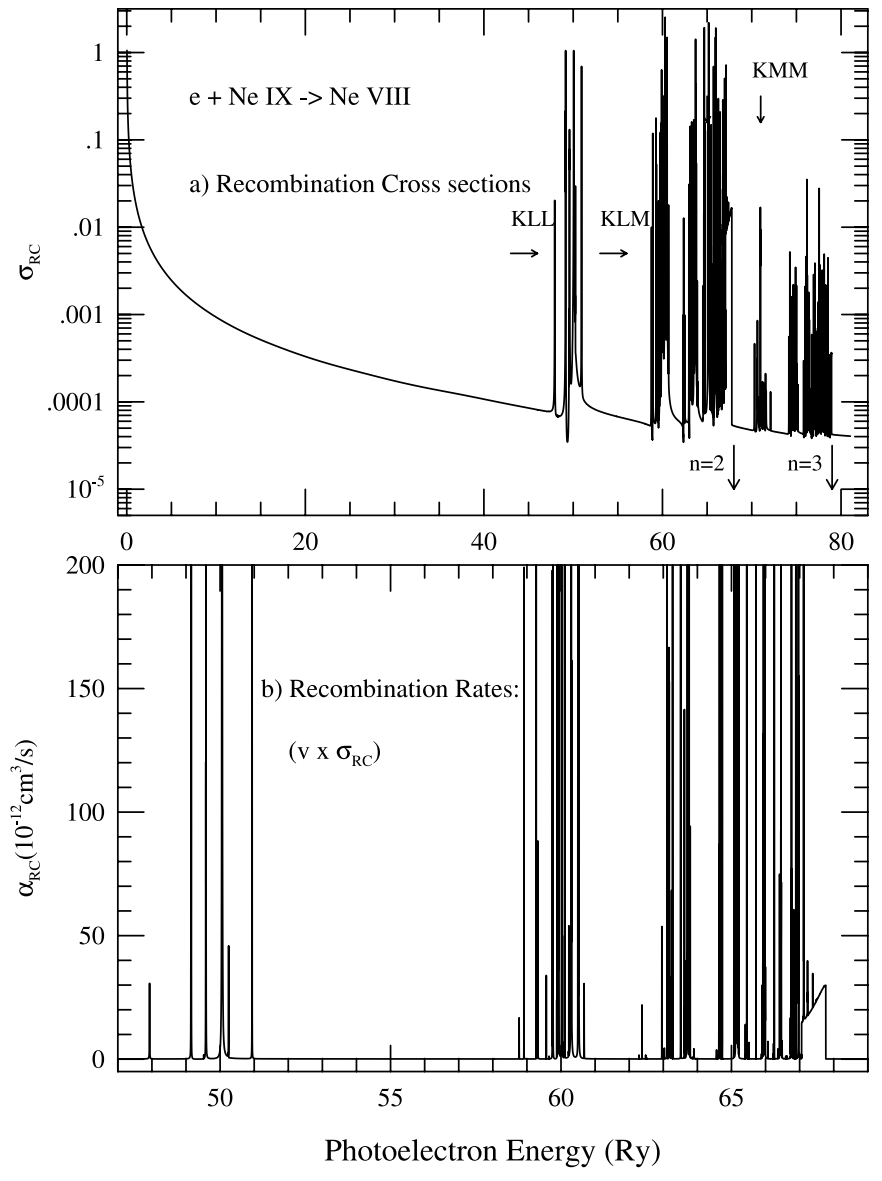

FIG. 3.-(a) Total unified (electron+ion) recombination cross sections, $\sigma_{\mathrm{RC}}$, and $(b)$ unified recombination rate coefficients, $\alpha_{\mathrm{RC}}(E)$, with photoelectron energy of Ne VIII. Separated resonance complexes, KLL, KLM, etc., of $n=2$ and $\mathrm{KMM}, \mathrm{KMN}$, etc., of $n=3$ thresholds can be noted; $\alpha_{\mathrm{RC}}(E)$, convolved with an energy bandwidth is a measurable quantity.

section summed over the contributions from channels with various target thresholds for ionization, and Figure $1 b$ presents the partial cross sections of the ground level ionized into the ground $1 s^{2}\left({ }^{1} S_{0}\right)$ level of residual ion Ne Ix. The resonances at high energies belong to the Rydberg series converging on to the $n=2,3$ levels of core ion Ne Ix. These are the well-known KLL, KLM resonances, for example, as discussed by Nahar \& Pradhan (2003). Since the first excited levels of $n=2$ thresholds of the core ion $\mathrm{Ne}$ Ix lie at high energies, the cross section decreases monotonically over a large energy range before the Rydberg series of resonances appear. The total and the partial cross sections are identical below the first excited level of the residual ion beyond which total $\sigma_{\mathrm{PI}}$ increases due to added contributions from excited channels (Fig. 1). A distinct difference between the total and partial cross sections in Figure 1 comes from the contribution of channels with excited $n=2$ thresholds. The $K$-shell ionization jump at the $n=2$ target levels in total $\sigma_{\mathrm{PI}}$ is due to inner-shell photoionization:

$$
h \nu+\mathrm{Ne} \operatorname{VIII}\left(1 s^{2} 2 s\right) \rightarrow e+\mathrm{Ne} \operatorname{Ix}(1 s 2 s, 1 s 2 p) .
$$

In X-ray photoionization models inner-shell edges play an important role in overall ionization rates.

Figure 2 presents partial photoionization cross sections of excited Rydberg series of levels $1 s^{2} n p, 2 \leq n \leq 7$ of Ne VIII. The figure illustrates the resonant structures at higher energies. especially the photoexcitation-of-core (PEC) resonances at energies 


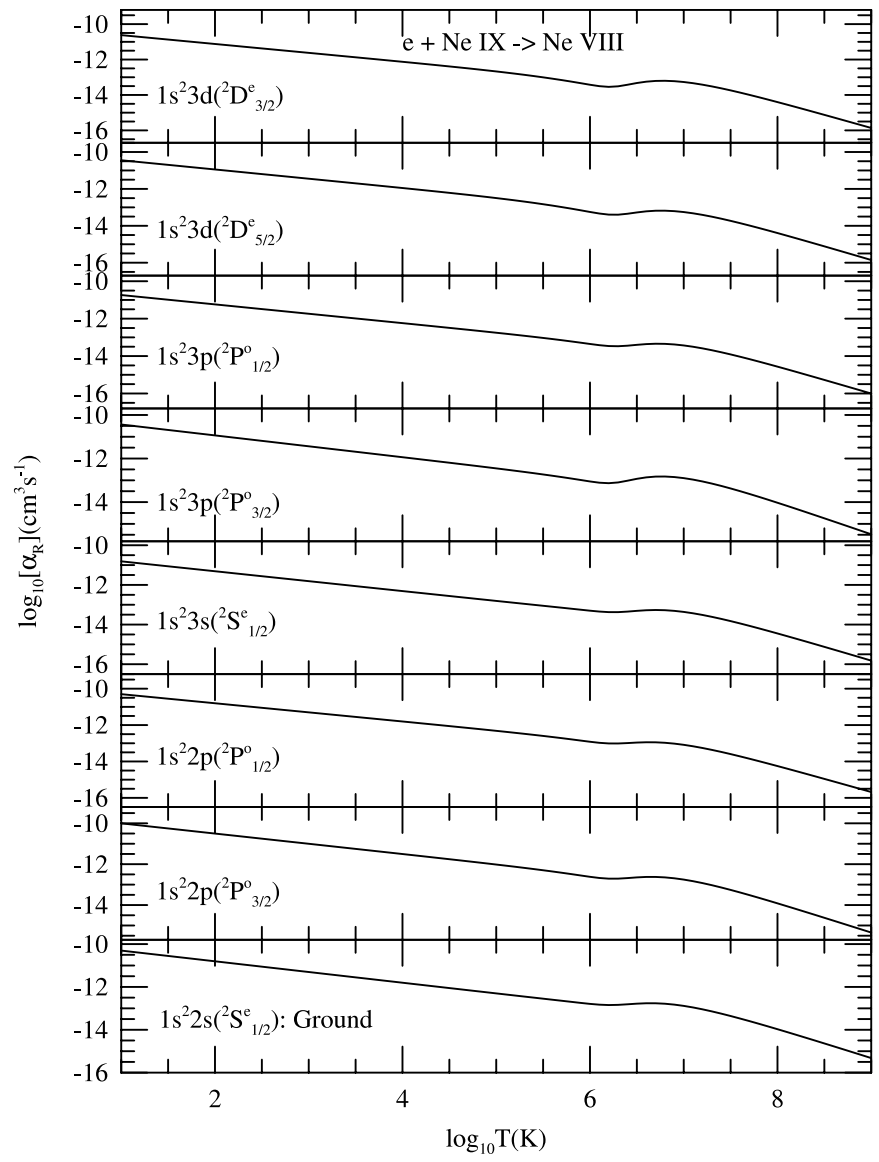

FIG. 4.-Recombination rate coefficients for $n=2$ and 3 levels of Ne vIII.

associated with dipole transitions in the core ion. PEC resonances exist in photoionization cross sections for all excited bound levels of Ne vII. They are seen in Figure 2 at photon energies 67.75 ryd due to core excitations to $n=2$ thresholds $1 s 2 p\left({ }^{3} P_{1}^{o}\right), 1 s 2 p\left({ }^{1} P_{1}^{o}\right)$, and at 78.9 ryd to $n=3$ thresholds $1 s 3 p\left({ }^{3} P_{1}^{o}\right)$, and $1 s 3 p\left({ }^{1} P_{1}^{o}\right)$. At these energies the core ion goes through a dipole allowed transition while the outer electron remains a "spectator" in a doubly excited resonance state, which is followed by autoionization into the ground level of the core ion. The PEC effect becomes increasingly prominent for cross sections of higher excited levels. The PEC resonances depict the nonhydrogenic behavior of cross sections of excited levels and correspond directly to the inverse process of DR.

\subsubsection{Electron-Ion Recombination}

We compute two related quantities for electron-ion recombination: the collision strength, $\Omega_{\mathrm{RC}}$, and the recombination cross sections, $\sigma_{\mathrm{RC}}$ (eqs. [10] and [11]). They show similar features; however, $\sigma_{\mathrm{RC}}$ diverges at zero photoelectron energy and therefore it is useful to compute $\Omega_{\mathrm{RC}}$. The total unified photorecombination cross section $\sigma_{\mathrm{RC}}$ for Ne VIII is presented in Figure $3 a$. The $\sigma_{\mathrm{RC}}$, like the photoionization cross sections $\sigma_{\mathrm{PI}}$, decays smoothly with energy until the emergence of resonance complexes at high energies. However, $\sigma_{\mathrm{RC}}$ is more complicated than a single-level $\sigma_{\text {PI }}$ since photoionization cross sections of all levels contribute to the total $\sigma_{\mathrm{RC}}$. A few resonance complexes are marked in the Figure $3 a-\mathrm{KLL}, \mathrm{KLM}, \mathrm{KLN}$, etc., converging onto the $n=2$ thresholds, and KMM, KMN, etc., converging onto the $n=3$ thresholds (KLL denotes the series $1 s 2 l 2 l$, KLM denotes $1 s 2 l 3 l^{\prime}$, etc.). These resonances manifest themselves as dielectronic

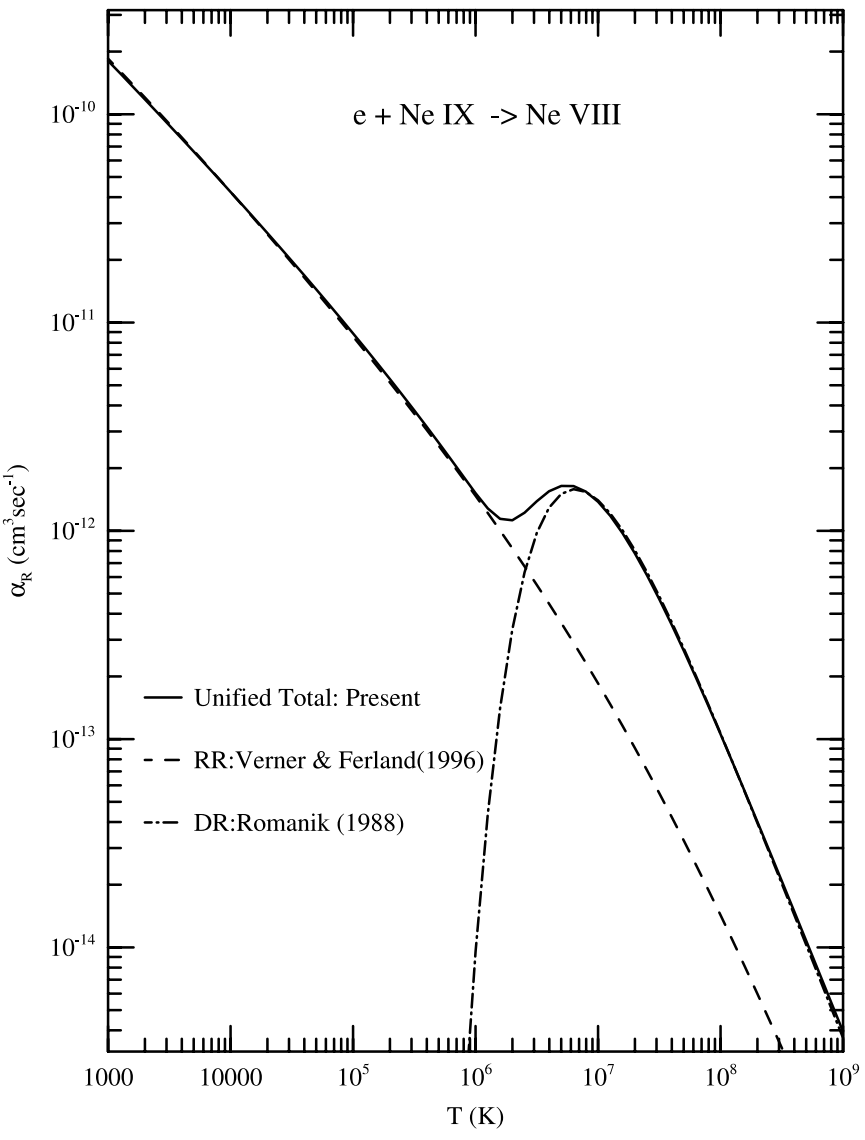

FIG. 5.-Total unified recombination rate coefficients, $\alpha_{R}(T)$, for Ne VIII (solid curve); RR rates (dashed curve) are by Verner \& Ferland (1996), and DR rates (dot-dashed curve) are by Romanik (1988).

satellite lines observed in tokamaks, electron-beam ion-traps (EBITs), ion storage rings, and high-temperature astrophysical sources. The KLL complexes are usually the most prominent and have been well studied in previous works (e.g., Gabriel 1972; Pradhan \& Zhang 1997; Zhang et al. 1999; Oelgoetz \& Pradhan 2001), for various ions.

We also calculate velocity- or energy-dependent photorecombination rates for comparison with experiments,

$$
\alpha_{\mathrm{RC}}(E)=v \sigma_{\mathrm{RC}}(E)
$$

where $v$ is the velocity and $E$ is the photoelectron energy, as measured in experiments. Figure $3 b$ presents the expanded part of the resonance series converging onto the $n=2$ thresholds in $\alpha_{\mathrm{RC}}(E)$ of $\mathrm{Ne}$ vIII. The observed shape corresponds to the detailed $\alpha_{\mathrm{RC}}(E)$, as shown in Figure $3 b$, convolved over the monochromatic bandwidth in the experiment (e.g., as described for C III in Pradhan et al. 2001).

Level-specific recombination rate coefficients $\alpha_{R}(T)$ of Ne VIII are presented for 98 levels. They correspond to all associated $J \pi$ levels $i \equiv n(S L J)$ with $n \leq 10$ and $\ell \leq 9$. Figure 4 presents $\alpha_{i}(T)$ into the eight lowest excited $n=2$ and 3 levels of $n(S L J)$ : $2 s^{2} S_{0}, 2 p{ }^{2} P_{1 / 2,3 / 2}^{o}, 3 s{ }^{2} S_{0}, 3 p{ }^{2} P_{1 / 2,3 / 2}^{o}$, and $3 d^{2} D_{3 / 2,5 / 2}$. These rates are relatively smooth except for a small and diffuse DR "bump."

Total recombination rates for a wide temperature range are given in Table 3. The main features are illustrated and compared with the available data for RR and DR rates in Figure 5. The BPRM total unified recombination rate coefficient $\alpha_{R}(T)$ (solid 


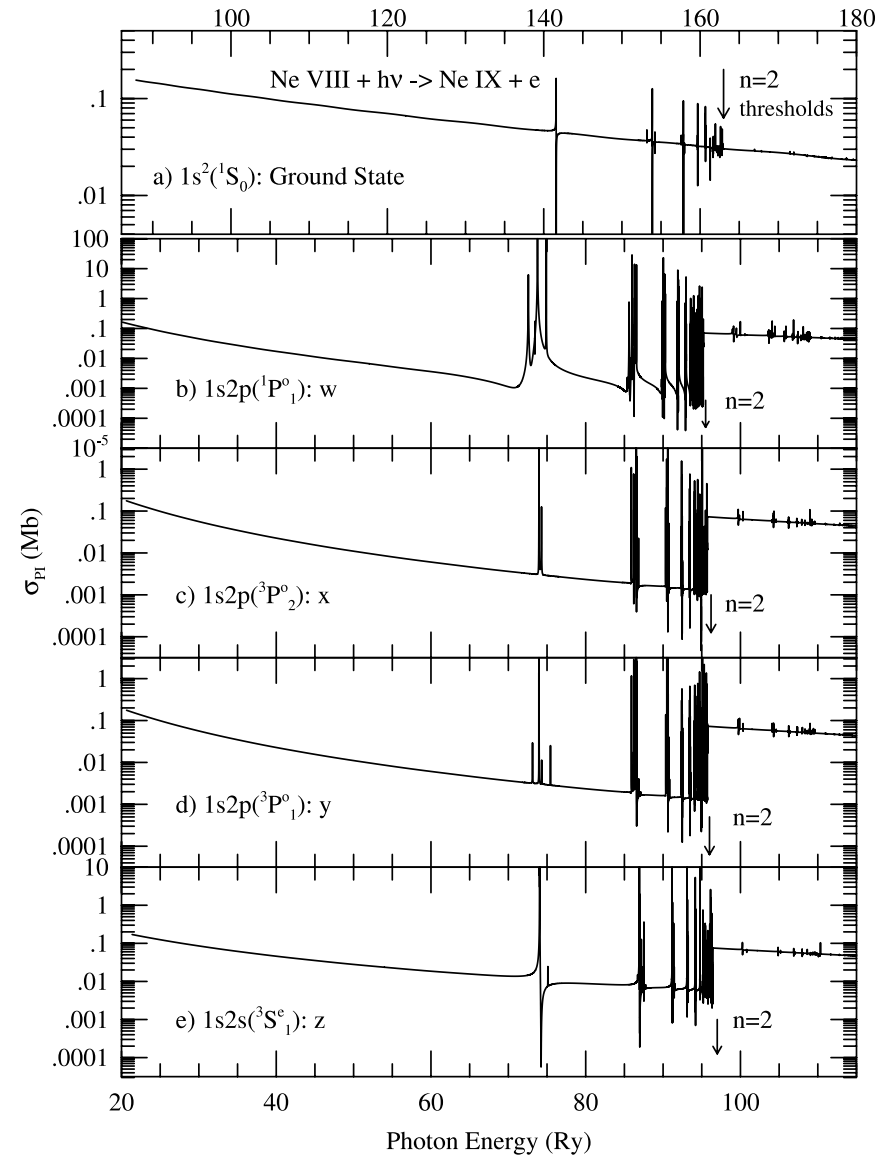

Fig. 6.- Level-specific photoionization cross sections of Ne Ix: $(a)$ the ground level, $1 s^{2}\left({ }^{1} S_{0}\right)$, and four excited $(b) 1 s 2 p\left({ }^{1} P_{1}^{o}\right),(c) 1 s 2 p\left({ }^{3} P_{2}^{o}\right),(d) 1 s 2 p\left({ }^{3} P_{1}^{o}\right)$, and (e) $1 s 2 s\left({ }^{3} S_{1}\right)$ levels corresponding to the prominent resonance $(w)$, intercombination $(y)$, and forbidden $(x, z)$ diagnostic $\mathrm{X}$-ray lines. $K$-shell ionization edge is noticed at the $n=2$ thresholds for the excited levels.

curve) shows the following typical features. It starts with a high value at very low temperatures due to the dominance of direct recombination (RR) into an infinite number of high- $n$ levels and decreases with increasing $T$ until high temperatures when there is a big rise due to appearance of resonances and the consequent dominance of DR. This is followed by a monotonic decay due to exponential damping in the assumed Maxwellian distribution of electrons.

The present total unified recombination rate coefficients for $\mathrm{Ne}$ VIII agree very well with the RR rate coefficients (dashed curve) by Verner \& Ferland (1996) and the DR rates (dot-dashed curve) by Romanik (1988). This is expected since the interference between RR and DR is small for Li-like ions, and hence the sum of independently calculated RR and DR rates should equal to that of the unified rates.

\subsection{Ne IX}

A total of 198 bound levels are found for Ne IX with $n \leq 10$, $0 \leq l \leq 9,0 \leq L \leq 14$, and total angular momentum of $0 \leq$ $J \leq 10$. In the following two subsections we describe the detailed results for photoionization and recombination for these levels.

\subsubsection{Photoionization}

Partial and total photoionization cross sections are presented for all 198 bound levels $(n \leq 10)$ of Ne Ix. Illustrative results are presented in Figure 6.
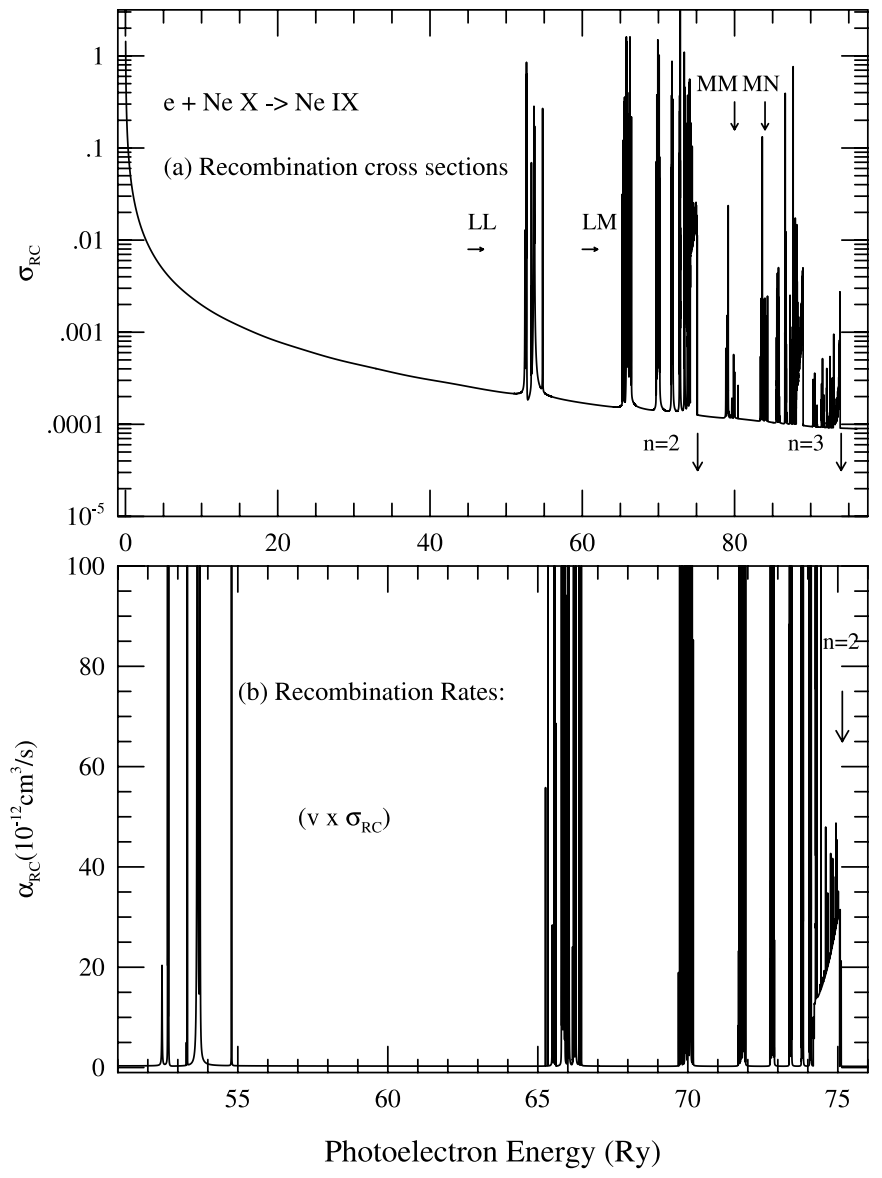

FIG. 7.-(a) Total unified (electron+ion) recombination cross sections, $\sigma_{\mathrm{RC}}$, and $(b)$ unified recombination rate coefficients, $\alpha_{\mathrm{RC}}(E)$ with photoelectron energy of Ne Ix. Note the separated resonance complexes, LL, LM, etc., of $n=2$ and MM, MN, etc., of $n=3$ and NN, NO, etc., of $n=4$ thresholds. The quantity $\alpha_{\mathrm{RC}}(E)$, convolved with a bandwidth, is measurable.

Figure 6 presents samples of level-specific photoionization cross section of Ne Ix. The ground level, $1 s^{2}\left({ }^{1} S_{0}\right)$, cross sections are in the topmost panel, while the lower four panels present $\sigma_{\mathrm{PI}}$ of the four lowest $n=2$ excited levels of Ne Ix. These excited levels correspond to the prominent X-ray lines of the $\mathrm{K} \alpha$ complex of He-like ions: the resonance line $\left[w: 1 s^{2}\left({ }^{1} S_{0}\right) \leftarrow\right.$ $\left.1 s 2 p\left({ }^{1} P_{1}^{o}\right)\right]$, the intercombination line $\left[y: 1 s^{2}\left({ }^{1} S_{0}\right) \leftarrow 1 s 2 p\left({ }^{3} P_{1}^{o}\right)\right]$, and the forbidden lines $\left[x: 1 s^{2}\left({ }^{1} S_{0}\right) \leftarrow 1 s 2 p\left({ }^{3} P_{2}^{o}\right)\right.$ and $z$ : $\left.1 s^{2}\left({ }^{1} S_{0}\right) \leftarrow 1 s 2 s\left({ }^{3} S_{1}\right)\right]$, respectively. These well-known lines are valuable as spectral diagnostics of temperature, density, ionization balance, and abundances in the plasma source.

In the photoionization cross section of the ground level $\left(1 s^{2}{ }^{1} S_{0}\right)$ of $\mathrm{Ne}$ Ix the Rydberg series of resonances, LL and $\operatorname{Ln}^{\prime}\left(n^{\prime}>2\right)$, begin at fairly high energies owing to the high $n=2$ excitation thresholds of $\mathrm{Ne}$ x. Whereas the ground level $\sigma_{\mathrm{PI}}$ of Ne Ix does not show a $K$-shell jump at the $n=2$ threshold, this is clearly seen in the level-specific photoionization cross sections for

$$
h \nu+\mathrm{Ne} \operatorname{Ix}(1 s 2 s, 1 s 2 p) \rightarrow e+\mathrm{Ne} \times(2 s, 2 p)
$$

in Figures $6 b-6 e$.

\subsubsection{Electron-Ion Recombination}

Total unified photorecombination cross section $\sigma_{\mathrm{RC}}$ for Ne IX is presented in Figure 7a. It corresponds to the summed contributions from photoionization cross sections of all levels, as 


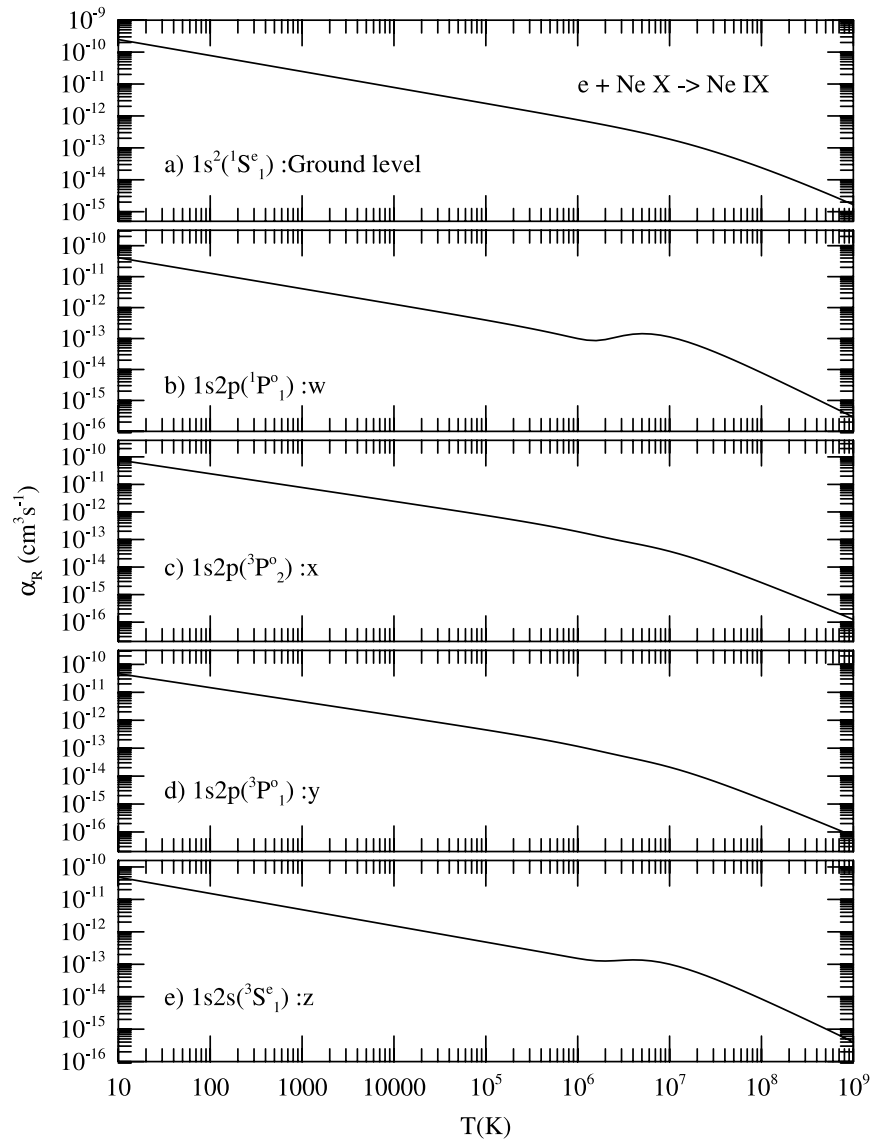

FIG. 8.-Level-specific recombination rate coefficients for $\mathrm{Ne}$ IX into the ground and excited $n=2$ levels responsible for the prominent X-ray lines $w$, $x, y$, and $z$.

well as the high- $n$ DR cross sections. The $\sigma_{\mathrm{RC}}$ decays smoothly with energy until resonance complexes appear at very high energies, as expected for a He-like ion. The Rydberg series of resonance complexes are LL, LM, LN, etc., converging onto the $n=2$ thresholds, MM, MN, etc., converging onto the $n=3$ thresholds, and NN, NO, etc., converging on to the $n=4$ thresholds; LL denotes $2 l 2 l^{\prime}$, LM denotes $2 l 3 l^{\prime}$, etc. The $n=4$ resonances are weaker and very narrow.

Figure $7 b$ presents an expanded part of the $n=2$ resonances in the velocity-dependent photorecombination rates $\left[\alpha_{\mathrm{RC}}(E)\right]$, shown as a function of the photoelectron energy. As mentioned before, $\left\langle v \alpha_{\mathrm{RC}}\right\rangle$ is the quantity measured in experiments, convolved over the monochromatic bandwidth of the beam.

Level-specific recombination rate coefficients are presented for 198 levels of $n(S L J)$ with $0 \leq J \leq 10$ and $n \leq 10$. Figure 8 presents level-specific recombination rates for the ground and the $n=2$ levels corresponding to the X-ray lines $w, x, y$, and $z$ of Ne Ix. The rates generally show a relatively smooth decay with temperature, but with a small but discernible rise due to DR resonances at high energies.

Total unified recombination rate coefficients are given in Table 3. The main features are illustrated and compared with the available RR and DR rates in Figure 9. The BPRM unified $\alpha_{R}(T)$ (solid curve) shows typical features similar to Ne vIII. The recombination rate coefficient is high at very low temperatures due to the dominance of RR into an infinite number of high- $n$ levels and decreases with increasing $T$ until at high temperatures where a "shoulder" is formed due to the dominance of DR, which is

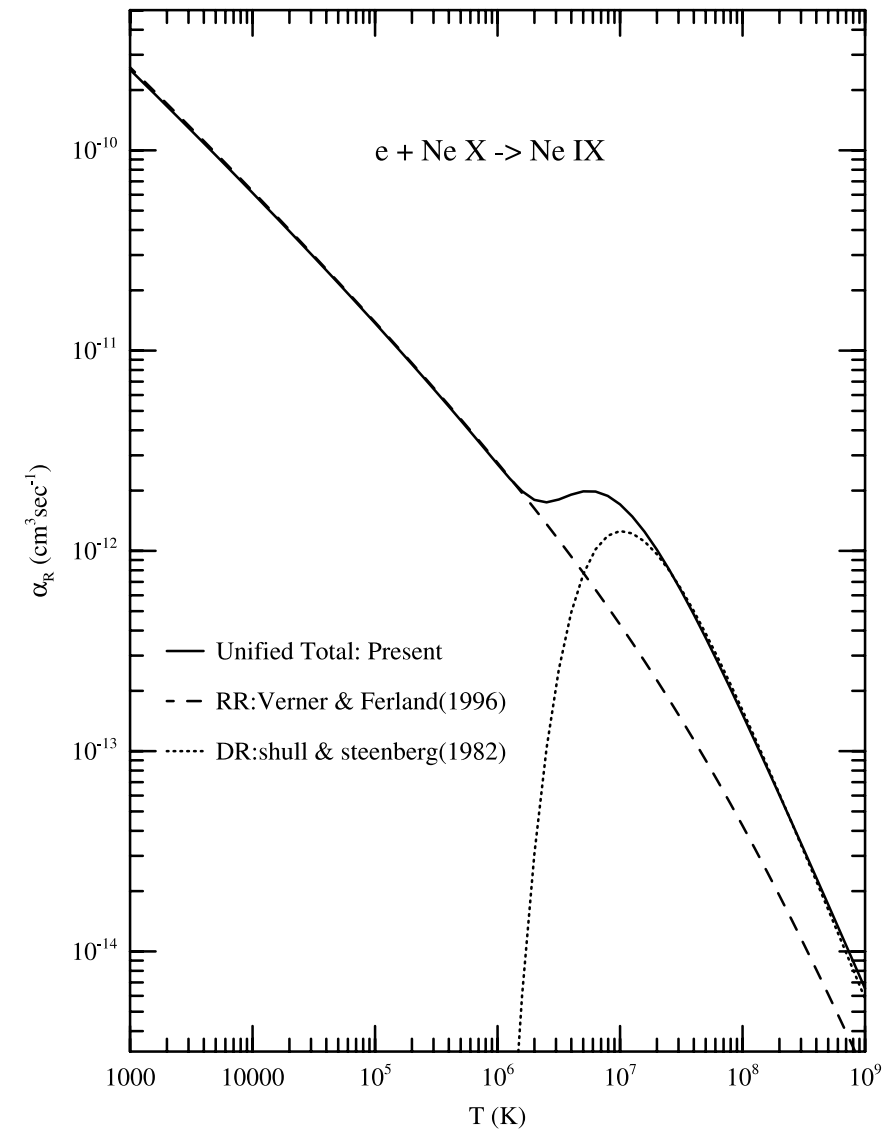

FIG. 9.- Total unified recombination rate coefficients, $\alpha_{R}(T)$, for $\mathrm{Ne}$ IX (solid curve). The RR rates (dashed curve) are by Verner \& Ferland (1996), and the DR rates (dotted curve) are by Shull \& Steenberg (1982).

followed by monotonic decay due to exponential damping of recombination cross sections by the Maxwellian electron distribution. The present total unified recombination rate coefficients, $\alpha_{R}(T)$, for Ne Ix compare very well with the RR rate coefficients (dashed curve) by Verner \& Ferland (1996), and the fits to DR rates (dotted curve) by Shull \& van Steenberg (1982), as might be expected for a simple atomic system.

\subsection{Dielectronic Satellite Intensities from Unified Recombination Cross Sections}

Dielectronic satellites are the resonances in unified recombination cross sections (e.g., Fig. 2). The present unified cross sections correspond directly to the experimentally measurable cross sections, or beam-velocity-averaged rate coefficients (e.g., Pradhan et al. 2001). The strong resonances are seen as dielectronic satellites. The computed profiles and intensities from the unified cross sections can be directly compared with measured quantities and observed recombination spectra. Whereas the unified cross sections include both the RR and DR contributions, the DR dominates at resonance (satellite) energies. Nevertheless, it is the total $\mathrm{RR}+\mathrm{DR}$ that is, in fact, always observed. While dielectronic satellite spectra of $\mathrm{He}$-like $\mathrm{Ne}$ IX are very weak, since the radiative decay rate of associated resonances is small compared to autoionization, we illustrate in principle how the dielectronic satellite intensities may be obtained in a straightforward manner.

Figure 10 shows an expanded view of the unified $\sigma_{\mathrm{RC}}(E)$ for the KLL "line" complex of recombination from He-like Ne Ix to Li-like Ne VIII. Unlike calculations in the individual resonance 


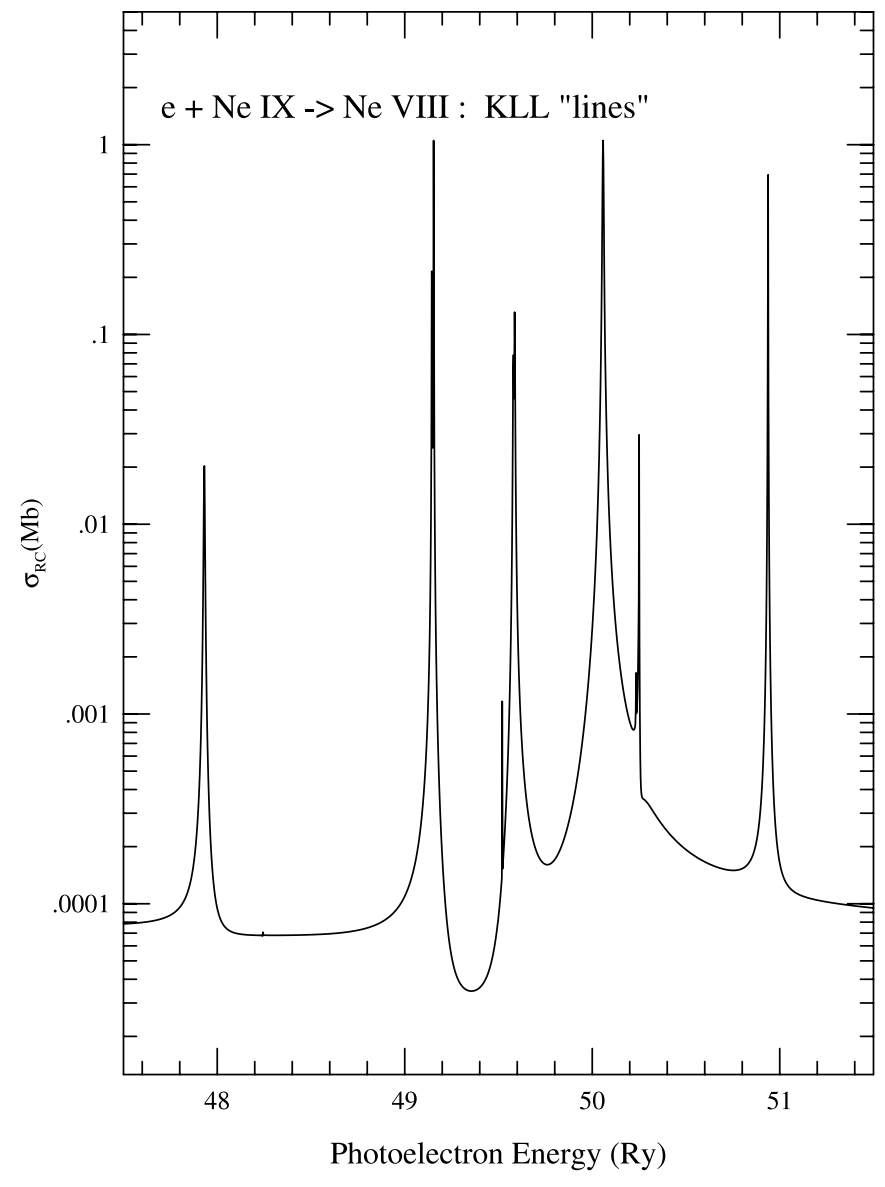

FIG. 10.-Expanded view of the KLL complex of "lines," dielectronic satellites (DESs) owing to autoionizing resonances that decay radiatively following dielectronic recombination of electrons with $\mathrm{Ne}$ IX to form $\mathrm{Ne}$ VIII. Individual features may be convolved with a Maxwellian to yield rate coefficients for dielectronic satellite intensities.

approximation, the profiles are obtained from the unified coupledchannel calculations in an ab initio manner and correspond to autoionization widths and intensities of the dielectronic satellites. Straightforward integration assuming a Maxwellian electron distribution over the resonances yields the dielectronic satellite rate coefficient. Generally, one refers to satellites as resonances in the observed recombination spectra relative to the "resonance" line owing to electron impact excitation of the first dipole transition in the ion (e.g., the " $w$ " line in He-like ions), i.e., the satellite-toresonance line ratio. The KLL complex in high- $Z$ He-like ions such as Fe xxv is well known and experimentally well resolved (e.g., Pradhan \& Zhang 1997 and references therein). For Ne IX, however, we simply report the total rate coefficients for the KLL and KLM dielectronic satellite complexes at a few temperatures close to the maximum abundance in coronal equilibrium in Table 4. Individual resonances are extremely narrow and not observable in astrophysical spectra. We emphasize, however, that theoretically there is no difficulty in resolving these satellites further if needed. The KLL and KLM rates behave differently with electron temperature owing to their respective positions on the energy scale. For low temperatures we find that the KLL rate exceeds that of KLM by up to 2 orders of magnitude. But as the temperature increases the KLM rate goes up and remains higher than the KLL rate by up to factors of a few. For Ne IX, therefore, the KLL/KLM ratios could conceivably be used as temperature diagnostics, provided they are observed and resolved.
TABLE 4

Recombinaton Rate Coefficients for SAtellite Lines of Ne VIII

\begin{tabular}{|c|c|c|}
\hline \multirow{2}{*}{$\begin{array}{c}\log _{10} T \\
(\mathrm{~K})\end{array}$} & \multicolumn{2}{|c|}{$\alpha_{R}\left(\mathrm{~cm}^{3} \mathrm{~s}^{-1}\right)$} \\
\hline & KLL & KLM \\
\hline 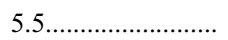 & $1.247 \mathrm{E}-21$ & $1.302 \mathrm{E}-23$ \\
\hline 5.6................ & $1.463 \mathrm{E}-19$ & $4.453 \mathrm{E}-21$ \\
\hline 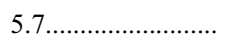 & $6.019 \mathrm{E}-18$ & $4.276 \mathrm{E}-19$ \\
\hline 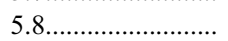 & $1.075 \mathrm{E}-16$ & $1.497 \mathrm{E}-17$ \\
\hline 5.9............................. & $9.897 \mathrm{E}-16$ & $2.350 \mathrm{E}-16$ \\
\hline 6.0.1. & $5.378 \mathrm{E}-15$ & $1.950 \mathrm{E}-15$ \\
\hline 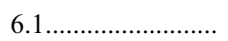 & $1.923 \mathrm{E}-14$ & $9.759 \mathrm{E}-15$ \\
\hline 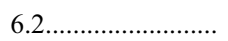 & $4.928 \mathrm{E}-14$ & $3.266 \mathrm{E}-14$ \\
\hline 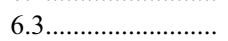 & $9.695 \mathrm{E}-14$ & $7.943 \mathrm{E}-14$ \\
\hline 6.4. & $1.546 \mathrm{E}-13$ & $1.499 \mathrm{E}-13$ \\
\hline 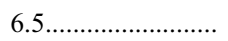 & $2.086 \mathrm{E}-13$ & $2.311 \mathrm{E}-13$ \\
\hline 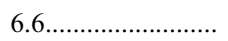 & $2.465 \mathrm{E}-13$ & $3.037 \mathrm{E}-13$ \\
\hline 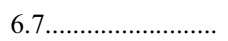 & $2.622 \mathrm{E}-13$ & $3.515 \mathrm{E}-13$ \\
\hline 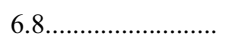 & $2.564 \mathrm{E}-13$ & $3.676 \mathrm{E}-13$ \\
\hline 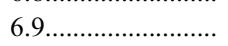 & $2.347 \mathrm{E}-13$ & $3.548 \mathrm{E}-13$ \\
\hline $7.0 \ldots \ldots \ldots \ldots \ldots \ldots$ & $2.038 \mathrm{E}-13$ & $3.213 \mathrm{E}-13$ \\
\hline 7.1 & $1.696 \mathrm{E}-13$ & $2.766 \mathrm{E}-13$ \\
\hline 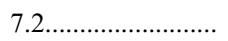 & $1.366 \mathrm{E}-13$ & $2.288 \mathrm{E}-13$ \\
\hline $7.3 \ldots$ & $1.071 \mathrm{E}-13$ & $1.832 \mathrm{E}-13$ \\
\hline 7.4.․․․․․․․…. & $8.224 \mathrm{E}-14$ & $1.431 \mathrm{E}-13$ \\
\hline $7.5 \ldots \ldots \ldots-1 . \cdots \cdots$ & $6.210 \mathrm{E}-14$ & $1.095 \mathrm{E}-13$ \\
\hline 7.6.................. & $4.628 \mathrm{E}-14$ & $8.246 \mathrm{E}-14$ \\
\hline 7.7 …………… & $3.412 \mathrm{E}-14$ & $6.131 \mathrm{E}-14$ \\
\hline 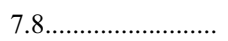 & $2.495 \mathrm{E}-14$ & $4.514 \mathrm{E}-14$ \\
\hline 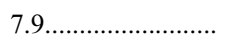 & $1.812 \mathrm{E}-14$ & $3.296 \mathrm{E}-14$ \\
\hline 8.0................................ & $1.310 \mathrm{E}-14$ & $2.392 \mathrm{E}-14$ \\
\hline 8.1.................................. & $9.422 \mathrm{E}-15$ & $1.727 \mathrm{E}-14$ \\
\hline $8.2 \ldots \ldots \ldots \ldots \ldots \ldots$ & $6.757 \mathrm{E}-15$ & $1.241 \mathrm{E}-14$ \\
\hline $8.3 \ldots \ldots \ldots \ldots \ldots \ldots$ & $4.833 \mathrm{E}-15$ & $8.898 \mathrm{E}-15$ \\
\hline 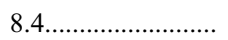 & $3.449 \mathrm{E}-15$ & $6.361 \mathrm{E}-15$ \\
\hline 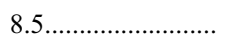 & $2.458 \mathrm{E}-15$ & $4.539 \mathrm{E}-15$ \\
\hline 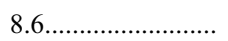 & $1.749 \mathrm{E}-15$ & $3.233 \mathrm{E}-15$ \\
\hline 8.7.......................... & $1.243 \mathrm{E}-15$ & $2.300 \mathrm{E}-15$ \\
\hline 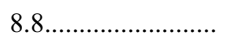 & $8.829 \mathrm{E}-16$ & $1.635 \mathrm{E}-15$ \\
\hline 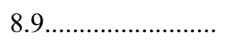 & $6.267 \mathrm{E}-16$ & $1.161 \mathrm{E}-15$ \\
\hline $9.0 \ldots$ & $4.446 \mathrm{E}-16$ & $8.239 \mathrm{E}-16$ \\
\hline
\end{tabular}

The $\boldsymbol{R}$-matrix method, based on the CC approximation, for unified (electron+ion) recombination yields essentially exact representation of dielectronic satellite spectra, as exemplified in Figure 10. The unified method provides a complete description of the natural (electron+ion) recombination process as observed through high-resolution instruments or measured in the laboratory. Resonances appear in an ab initio manner from the coupling among open and closed channels in the wave function expansion in the unified method (Nahar \& Pradhan 2004). It is therefore fundamentally different from previous methods, largely based on the distorted wave and the isolated resonance approximations, which may obtain resonance structures perturbatively by considering resonances as a single bound state coupled to a continuum channel. As such, the unified method includes interference effects that may manifest themselves in overlapping resonances in complex cases, although for highly charged ions the differences may not be apparent.

A comparison of the present KLL intensities in Table 4 with those of Vainshtein \& Safronova (1978) show excellent agreement. For example, at $T=2 \times 10^{6} \mathrm{~K}$ the total $I(\mathrm{KLL}) /$ $I($ resonance $)=0.18$, in exact agreement with their ratio, and at $T=4 \times 10^{6} \mathrm{~K}$ the difference is about $5 \%$, where the resonance 
transition $1 s^{2}\left({ }^{1} S_{0}\right)-1 s 2 p\left({ }^{1} P_{1}^{o}\right)$ is the dipole transition in the He-like core Ne Ix. More detailed comparisons, also with experimental data (e.g., Wargelin et al. 2001), are presented in a separate publication (S. N. Nahar \& A. K. Pradhan 2006, in preparation), including satellite intensities of ions of potentially greater practical interest such as $\mathrm{Fe}$ xxv and Ni xxviI using the unified (electron+ion) recombination cross sections computed recently in this series.

\section{CONCLUSION}

Extensive results from relativistic calculations for total and level-specific photoionization and recombination cross sections and rate coefficients are presented for Li-like and He-like Ne VIII and Ne Ix. These should be of general interest in UV and X-ray spectroscopy of laboratory and astrophysical sources.

The present level-specific data can be used to construct recombination-cascade matrices for $\mathrm{Ne}$ VIII and $\mathrm{Ne}$ IX, to obtain effective recombination rates into specific fine-structure levels $n$ $(S L J)$ with $n \leq 10$ and $\ell \leq n-1$ (e.g., Pradhan 1985). Present total unified recombination rates agree very well with the sum of RR and DR of previous calculations, as expected for the He- and Li-like ions since RR and DR interference is small. The unified method for (electron+ion) recombination explicitly provides level-specific rates of hundreds of levels, and self-consistent photoionization cross sections and recombination rates, of many bound levels. The present data are more than sufficient for extrapolation to the high $n$ and $\ell$ necessary to account for all cascade contributions.

The method for computing dielectronic satellite intensities using unified recombination cross sections described in this work will be applied to more highly charged He-like ions.

The complete set of available data from these extensive calculations includes (1) photoionization cross sections, both total and partial, for bound fine-structure levels of $\mathrm{Ne}$ VIII and $\mathrm{Ne}$ IX up to $n=10$; (2) total unified recombination rate coefficients for $\mathrm{Ne}$ VIII and $\mathrm{Ne}$ IX, and level-specific recombination rate coefficients for levels up to $n=10$; (3) total unified recombination cross sections, and velocity-averaged rate coefficients for comparison with experiments in the usual low-energy region for $\mathrm{Ne}$ VIII and $\mathrm{Ne}$ Ix. Further calculations for other He-like and Li-like ions are in progress. All photoionization and recombination data are available electronically from the first author.

This work was supported partially by the NASA Astrophysical Theory Program. The computational work was carried out on the Cray SV1 at the Ohio Supercomputer Center in Columbus, Ohio.

\section{REFERENCES}

Bell, R. H., \& Seaton, M. J. 1985, J. Phys. B, 18, 1589

Berrington, K. A., Burke, P. G., Butler, K., Seaton, M. J., Storey, P. J., Taylor, K. T., \& Yu Yan. 1987, J. Phys. B, 20, 6379

Berrington, K. A., Eissner, W., \& Norrington, P. H. 1995, Comput. Phys. Commun., 92, 290

Canizares, C. R., et al. 2000, in Atomic Data Needs in X-Ray Astronomy, ed. M. A. Bautista, T. R. Kallman, \& A. K. Pradhan (NASA/CP-2000-209968), http://heasarc.gsfc.nasa.gov/docs/heasarc/atomic/proceed.html

Eissner, W., Jones, M., \& Nussbaumer, H. 1974, Comput. Phys. Commun., 8, 270

Gabriel, A. H. 1972, MNRAS, 160, 99

Gabriel, A. H., \& Jordan, C. 1969, Nature, 221, 947

Hummer, D. G., Berrington, K. A., Eissner, W., Pradhan, A. K., Saraph, H. E., \& Tully, J. A. 1993, A\&A, 279, 298

Lee, J. C., Ogle, P. M., Canizares, C. R., Marshall, H. L., Schulz, N. S., Morales, R., Fabian, A. C., \& Iwasawa, K. 2001, ApJ, 554, L13

Leighly, K. M., O’Brien, P. T., Edelson, R., George, I. M., Malkan, M. A., Matsuoka, M., Mushotzky, R. F., \& Peterson, B. M. 1997, ApJ, 483, 767

Mannervik, S., Dewitt, D., Engström, L., Lidberg, J., Lindroth, E., Schuch, R., \& Zong, W. 1998, Phys. Rev. Lett., 81, 313

Nahar, S. N. 1996, Phys. Rev. A, 53, 2417 2002, A\&A, 389, 716

2005 , ApJS, 158, 80

Nahar, S. N., \& Pradhan, A. K. 1992, Phys. Rev. Lett., 68, 1488

1994, Phys. Rev. A, 49, 1816

1997, ApJS, 111, 339 (Paper I)
Nahar, S. N., \& Pradhan, A. K. 2000, Phys. Scr., 61, 675 2003, ApJS, 149, 239

2004, Radiat. Phys. Chem., 70, 323

Nahar, S. N., Pradhan, A. K., \& Zhang, H. L. 2000, ApJS, 131, 375 2001, ApJS, 133, 255

Ness, J.-U., Brickhouse, N. S., Drake, J. J., \& Huenemoerder, D. P. 2003, ApJ, 598,1277

Oelgoetz, J., \& Pradhan, A. K. 2001, MNRAS, 327, L42

. 2004, MNRAS, 354, 1093

Pradhan, A. K. 1985, ApJ, 288, 824

Pradhan, A. K., Chen, G. X., Nahar, S. N., \& Zhang, H. L. 2001, Phys. Rev. Lett., 87, 3201

Pradhan, A. K., \& Zhang, H. L. 1997, J. Phys. B, 30, L571

Romanik, C. 1988, ApJ, 330, 1022

Sakimoto, K., Terao, M., \& Berrington, K. A. 1990, Phys. Rev. A, 42, 291

Scott, N. S., \& Burke, P. G. 1980, J. Phys. B, 13, 4299

Scott, N. S., \& Taylor, K. T. 1982, Comput. Phys. Commun., 25, 347

Schippers, S., Muller, A., Gwinner, G., Linkemann, J., Saghiri, A., \& Wolf, A. 2001, ApJ, 555, 1027

Seaton, M. J. 1987, J. Phys. B, 20, 6363

Shull, J. M., \& van Steenberg, M. 1982, ApJS, 48, 95

Vainshtein, L. A., \& Safronova, U. I. 1978, At. Data Nucl. Data Tables, 21, 49

Verner, D. A., \& Ferland, G. 1996, ApJS, 103, 467

Wargelin, B. J., Kahn, S. M., \& Beiersdorfer, P. 2001, Phys. Rev. A, 63, 2710

Zhang, H. L., Nahar, S. N., \& Pradhan, A. K. 1999, J. Phys. B, 32, 1459

Zhang, H. L., \& Pradhan, A. K. 1997, Phys. Rev. Lett., 78, 195 